\title{
1 RosettaSurf - a surface-centric computational design approach
}

2 Author list:

3 Andreas Scheck ${ }^{1,2}$, Stéphane Rosset ${ }^{1,2}$, Michaël Defferrard ${ }^{3}$, Andreas Loukas ${ }^{3}$, Jaume Bonet ${ }^{1,2}$,

4 Pierre Vandergheynst ${ }^{3}$, Bruno E Correia ${ }^{1,2^{*}}$

5 Affiliations:

61 Institute of Bioengineering, École Polytechnique Fédérale de Lausanne, Lausanne CH-1015,

7 Switzerland. 2 Swiss Institute of Bioinformatics (SIB), Lausanne CH-1015, Switzerland. 3 Signal

8 Processing Laboratory (LTS2), École Polytechnique Fédérale de Lausanne, Lausanne CH-1015,

9 Switzerland.

11 Proteins are typically represented by discrete atomic coordinates providing an accessible framework to describe different conformations. However, in some fields proteins are more accurately represented as near-continuous surfaces, as these are imprinted with geometric

14 (shape) and chemical (electrostatics) features of the underlying protein structure. Protein surfaces are dependent on their chemical composition and, ultimately determine protein function, acting as the interface that engages in interactions with other molecules. In the past, such

17 representations were utilized to compare protein structures on global and local scales and have shed light on functional properties of proteins. Here we describe RosettaSurf, a surface-centric computational design protocol, that focuses on the molecular surface shape and electrostatic

20 properties as means for protein engineering, offering a unique approach for the design of proteins

21 and their functions. The RosettaSurf protocol combines the explicit optimization of molecular

22 surface features with a global scoring function during the sequence design process, diverging

23 from the typical design approaches that rely solely on an energy scoring function. With this

24 computational approach, we attempt to address a fundamental problem in protein design related 
25 to the design of functional sites in proteins, even when structurally similar templates are absent

26 in the characterized structural repertoire. Surface-centric design exploits the premise that

27 molecular surfaces are, to a certain extent, independent of the underlying sequence and

28 backbone configuration, meaning that different sequences in different proteins may present

29 similar surfaces. We benchmarked RosettaSurf on various sequence recovery datasets and

30 showcased its design capabilities by generating epitope mimics that were biochemically validated.

31 Overall, our results indicate that the explicit optimization of surface features may lead to new 32 routes for the design of functional proteins.

\section{Keywords}

35 Molecular surface, surface similarity, computational protein design, protein-protein interactions, Rosetta

\section{Author Summary}

39 Finely orchestrated protein-protein interactions are at the heart of virtually all fundamental cellular

processes. Altering these processes or encoding new functions in proteins has been a long-

41 standing goal in computational protein design.

42 Protein design methods commonly rely on scoring functions that seek to identify amino acid

43 sequences that optimize structural configurations of atoms while minimizing a variety of physics-

44 based and statistical terms. The objectives of the large majority of computational design protocols

45 have been focused on obtaining a predefined structural conformation. However, routinely

46 introducing a functional aspect on designer proteins has been more challenging.

47 Our results suggest that the molecular surface features can be a useful optimization parameter

48 to guide the design process towards functional surfaces that mimic known protein binding sites

49 and interact with their intended targets. Specifically, we demonstrate that our design method can 
optimize experimental libraries through computational screening, creating a basis for highly

51 specific protein binders, as well as design a potent immunogen that engages with site-specific

52 antibodies. The ability to create proteins with novel functions will be transformative for biomedical

53 applications, providing many opportunities for the design of novel immunogens, protein

54 components for synthetic biology, and other protein-based biotechnologies.

57 Proteins are key components in living cells, performing many functions that commonly rely on

58 physical interactions between them and other molecules. Steady progress in structural biology

59 has unveiled the structures of a wide variety of proteins and their mode of interaction with other

60 molecular partners. High-resolution protein structures are studied at the atomic level, focusing on

61 the interactions between residues, backbone conformations, or the topology of secondary

62 structure elements, among others (1). The molecular surface arising from the three-dimensional

63 arrangement of the many atoms that compose a protein is, ultimately, determinant for protein

64 function and is therefore crucial for biological processes (2).

65 While discrete atomic-level protein representations have been invaluable for our understanding

66 of protein function, near-continuous surface-based representations offer the opportunity to study

67 protein structures using a different subset of features (e.g. electrostatic potentials, geometry).

68 In 1971, Lee and Richards introduced the concept of solvent-accessible surfaces, which in

69 practice are generated by rolling a probe approximating a solvent molecule over the protein atoms

70 (3). The molecular surface, often denoted as solvent-excluded surface or Connolly surface (4-6),

71 consists of the surface that can be directly contacted by the probe and the reentrant surface which

72 smooths over gaps between the atoms that were not accessible to the probe sphere $(5,7,8)$.

73 Numerical representations of surfaces have also been developed, ranging from dot surfaces, to

74 voxel representations and graphs (1,2,9-12). These representations allow the mapping of 
75 molecular and geometric properties onto the generated surface, including physicochemical

76 properties (such as electrostatics and hydrophobicity), and geometrical features (e.g. protrusions

77 or cavities) $(1,7,13)$. Having these features of proteins encoded in their surfaces facilitates

78 powerful computational analysis. Recently, the machine learning-based approach MaSIF

79 (molecular surface interaction fingerprinting) showcased the extraction of surface features from

80 molecular surfaces to study protein structure-function relationships $(14,15)$.

82 Intuitively, the molecular surface forms the boundary of the protein and its surroundings, thus

83 acting as the interface that engages in interactions with other molecules. The study of protein

84 structures as near-continuous molecular surfaces is therefore important to understand structural

85 and functional aspects of proteins, which may not be fully captured by a discrete atomic

86 representation $(13,16)$. A widely studied category of features of molecular surfaces is their

87 chemistry, in particular electrostatic potentials, and their implications for protein function. Most

88 notably, such representations have been used to study various types of protein-substrate

89 interactions $(17,18)$. However, many different aspects of proteins can be investigated with this

90 approach, including the solvation energies of proteins in different media, redox potentials, or

91 catalytic rates of enzymes (17). Other important metrics used to study molecular surfaces are

92 their shape-derived properties, commonly focusing on shape complementarity $\left(\mathrm{S}_{\mathrm{C}}\right)$ or shape

93 similarity $\left(\mathrm{S}_{\mathrm{S}}\right)$. Shape complementarity is frequent in the study of molecular recognition, e.g.

94 protein-protein or protein-ligand interactions. In particular, protein-protein interactions (PPIs) have

95 been extensively studied in terms of complementarity, showing that protein-protein interfaces are

96 often highly complementary, both in shape and in charge $(19,20)$. Moreover, shape

97 complementarity has been incorporated into protein docking to guide the prediction of protein

98 complexes (21-25) and to match binding pockets with potential ligands (26). On the other hand,

99 shape similarity has been used to globally and locally compare biomolecules, aiding the functional

100 annotation of proteins that show structural similarities but lack detectable sequence homology 
$101(11,27)$. Thus, shape similarity allows for the classification of local surface patches as well as the

102 prediction of ligand binding sites by pocket shape similarity $(1,11)$.

103 An important extension of such successful applications in analysis and prediction tasks using

104 surface-based representations is that of protein design (7), where the objective is to guide the

105 sequence search process to optimize specified surface features.

106 Here we present a new, surface-centric computational design strategy, termed RosettaSurf, that

107 uses a description of the molecular surface shape and electrostatic properties as objective

108 function for scoring optimization in protein design. Working at the surface-level of protein

109 structures offers a unique approach for the design of proteins and their functions, as molecular

110 surfaces are, to some extent, independent of the underlying sequence $(11,14)$. This means that

111 different sequences in different proteins may present similar surfaces $(11,14)$ - a premise that

112 drives our proposed methodological approach. In contrast, the large majority of computational

113 design workflows entail a discrete atomistic description of the proteins and sequence design is

114 typically performed on the atom arrangements and interactions; i.e. by sampling different amino

115 acid side chains and adjusting atoms in the protein structure to optimize a given energy function.

116 While these strategies have been successfully applied to design novel protein topologies (28-

11730 ), the design of functional proteins based solely on computational calculations remains a

118 challenge (31-38). The introduction of function into a designer protein often requires the

119 transplantation of functional sites by means of protein grafting (39-44). A major limitation of this

120 approach is the dependence on the identification of appropriate protein scaffolds (35). Therefore

121 the ability to accurately design surface patches could represent an alternative route to

122 functionalize computationally designed proteins.

123 RosettaSurf is a protocol implemented in the Rosetta software package (45) and we assessed its

124 performance with several benchmark tests, demonstrating the protocol's capabilities through the

125 recovery of protein interfaces and its application to functional protein design. 
127 In our benchmark studies we sought to compare the developed surface-centric design protocol,

128 RosettaSurf, to state-of-the-art macromolecular design approaches implemented in Rosetta. The

129 Rosetta energy function has been parametrized to evaluate and optimize the energy of many

130 different aspects of molecular interactions (e.g. protein stability, protein-ligand, protein-protein

131 and protein-nucleic acids, etc.); it contains both statistical and physics-based terms, being

132 calibrated using a discrete atomic representation of the molecules. This type of optimization has

133 been particularly successful for the design of novel sequences that fold into defined protein

134 structures. However, to design proteins that display defined motifs which can perform biological

135 functions has proven to be difficult. By focusing the design process on the areas where molecular

136 interactions occur - the protein surfaces - novel approaches attempting to design function into

137 proteins may represent new routes to address this problem.

138 Two types of metrics in surface comparison are considered: surface complementarity and surface

139 similarity. We show how the surface similarity score captures features of individual residues and

140 demonstrate the ability of the RosettaSurf protocol to recover amino acid sequences of native

141 protein interfaces. Furthermore, we highlight the performance of RosettaSurf for the design of

142 surface patches at protein interfaces.

\section{Single amino acid recovery}

145 To evaluate the accuracy of our design protocol, we performed a benchmark on the recovery of

146 single amino acids in native protein interfaces. The amino acid of interest is substituted by each

147 of the 19 amino acid identities (excluding cysteine) and the surfaces of the substituted rotamers

148 are compared to that of the native rotamer (Fig. 1A). The energy computed by Rosetta, Rosetta

149 Energy Unit (REU), serves as a baseline for comparison to the surface-centric design strategy.

150 We evaluated the recovery of the different amino acids for mutations made in the bound and 
151 unbound state of the protein complex, respectively (Fig. 1B). The recovery of an amino acid is

152 deemed successful when the native amino acid has the best score among all 19 amino acids and

153 is uniquely identifiable. In this benchmark we evaluated the performance of shape similarity,

154 electrostatic similarity and both combined in a surface similarity score. The surface similarity

155 measurement is highly accurate in identifying native amino acids in the bound state of the protein

156 complexes, showing consistently higher sequence recovery rates than Rosetta energy (Fig. 1B).

157 Incorporating the electrostatic similarity term into the surface score generally results in a boost in

158 recovery rates over shape similarity alone, in particular for amino acids that have close structural

159 doubles but differ in chemical properties, e.g. glutamine and glutamate, as well as, asparagine

160 and aspartate (Fig. 1B).

161 The Rosetta energy function shows the best recovery rates for amino acids with unique features

162 on their side-chains, i.e. glycine and proline. However, even for these cases, surface similarity

163 outperforms the recovery by REU. A similar trend can be observed for the unbound complexes,

164 although the general success of retrieving the native amino acid decreases for certain amino

165 acids. This trend is most obvious for polar residues and large side chains that have access to a

166 variety of different rotamer conformations as the lack of the binding partner allows a larger

167 conformational space for different rotamers to be explored.

168

169 Moreover, the surface similarity scores retrieved from an all-against-all amino acid comparison 170 demonstrate the high accuracy by which most amino acids can be identified (Fig. 1C). Here, we 171 computed mean surface similarity values for all substitutions tested, i.e. for each native amino 172 acid across the 100 protein complexes we computed the similarity of all other 19 amino acids to 173 the native one on average. The results show that each of the 19 amino acids is generally most 174 similar to itself, demonstrating that the method can accurately distinguish between the different 175 amino acid types. Notable exceptions are residues that share similar geometrical features, e.g. 176 phenylalanine and histidine or tyrosine. 
bioRxiv preprint doi: https://doi org/10.1101/2021.06.16.448645; this version posted June 16, 2021. The copyright holder for this preprint (which was not certified by peer review) is the author/funder, who has granted bioRxiv a license to display the preprint in perpetuity. It is made available under aCC-BY 4.0 International license.

177 Close inspection of the considered surface of a single amino acid demonstrates the local precision

178 of the surface similarity score (Fig. S2). Small off-rotamer deviations are captured on the point

179 cloud and are specific for the mismatching surface area. These results indicate that the surface

180 similarity score is sensitive to small differences in the comparison of two surface point clouds.

181 Overall, these results clearly show that the developed surface similarity score can capture local

182 differences in surfaces providing the basis for the evaluation of differences between full surface 183 patches. 
bioRxiv preprint doi: https://doi.org/10.1101/2021.06.16.448645; this version posted June 16, 2021. The copyright holder for this preprint (which was not certified by peer review) is the author/funder, who has granted bioRxiv a license to display the preprint in perpetuity. It is made available under aCC-BY 4.0 International license.

A

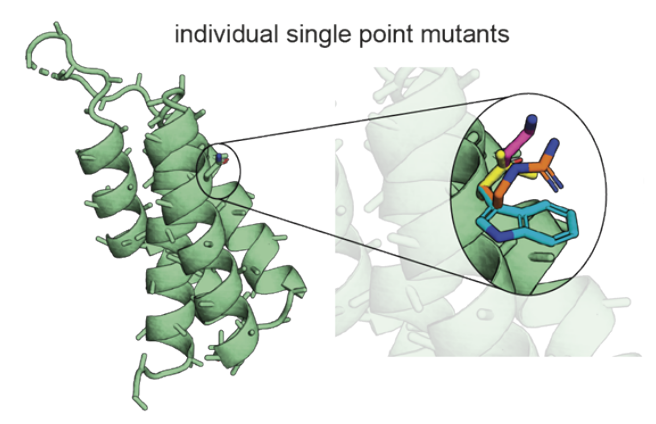

B
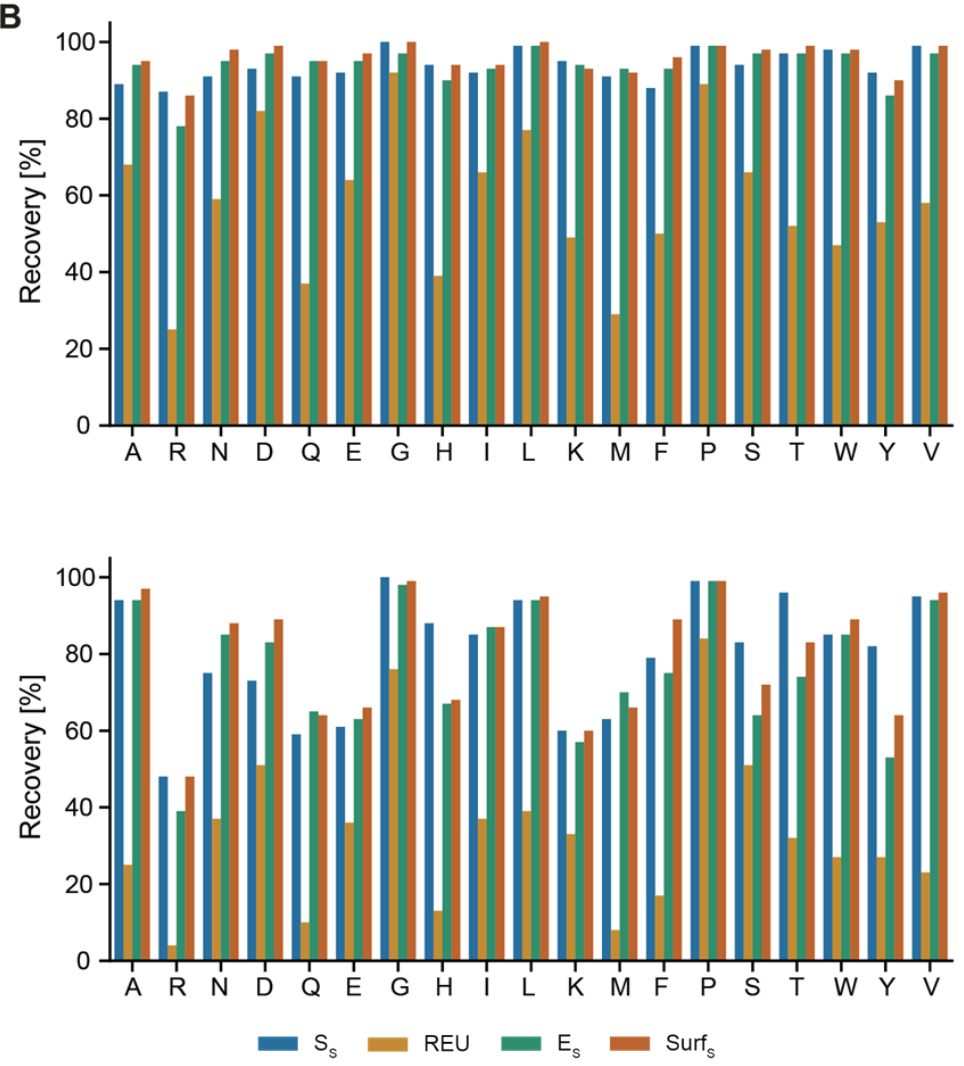
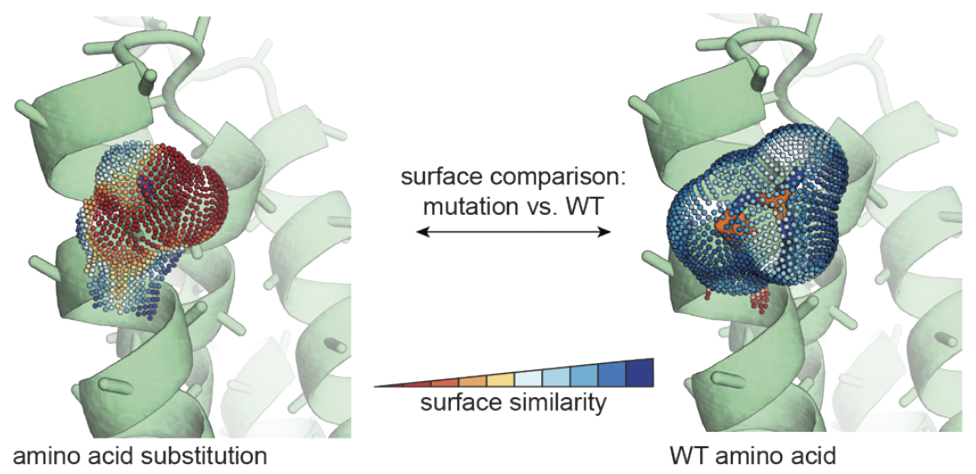

C
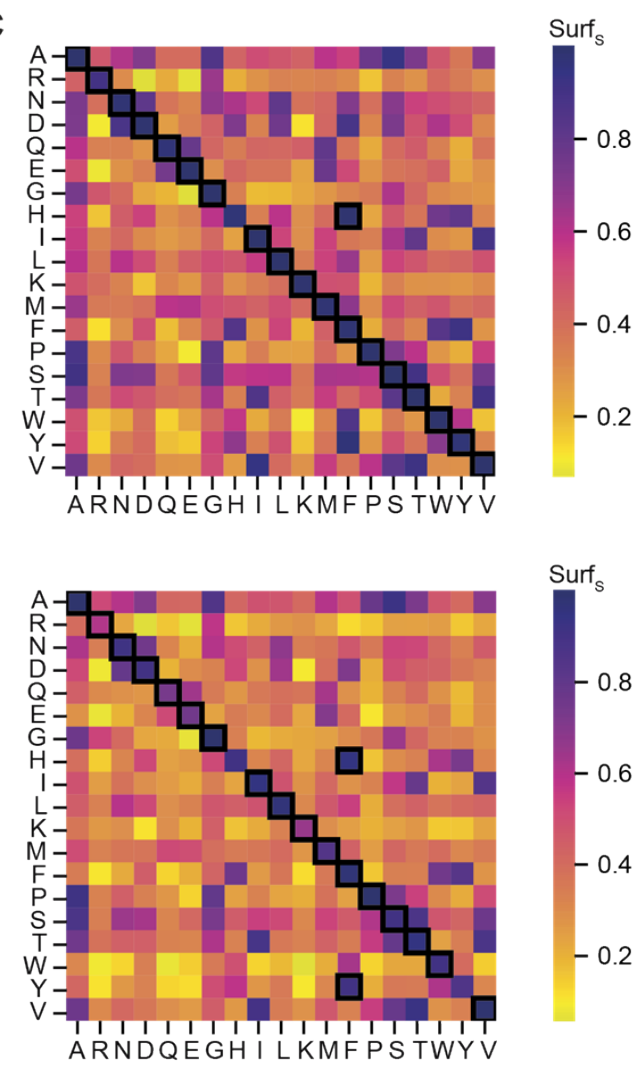

Figure 1. Single mutant discrimination using surface similarity score in protein-protein complexes. A) Surface

similarity evaluation protocol for single amino acids. B) Recovery for all 19 considered amino acid types in bound (top) is highlighted. 


\section{Protein interface sequence recovery}

193 Having shown that the implemented surface similarity score is sufficiently accurate to recover

194 individual amino acids, the following benchmark expands on recovering entire surface patches in 195 protein interfaces. We evaluated the ability of our surface-based sequence design protocols 196 (RosettaSurf and RosettaSurf-site) to recover natural protein interfaces as a gateway for the 197 design of proteins endowed with biochemical function. By focusing on protein interfaces, the 198 correct sequence does not solely depend on minimizing the Rosetta scoring function, but rather 199 needs to represent the surface properties of the interface site. While such design scenario has 200 limited applicability for the de novo design of protein-protein interactions, it is important for 201 applications in the domain of immunogen design for vaccine development where the surface 202 mimicry of known surfaces (neutralizing epitopes) is critical for the biological activity of this type 203 of design (34,36-38).

204 In this benchmark we considered nine protein-protein complexes, grouped into three categories 205 (low complementarity, high complementarity, antibody/antigen), and evaluated the performance 206 by assessing sequence recovery (Fig. 2A). We compared three different design approaches:

1) Standard Rosetta design (FixBB)

2) Surface-centric design (RosettaSurf)

3) Single-site-scanning surface-centric design (RosettaSurf-site)

210 All protocols operate on fixed backbones of the protein complexes that are presented in the native

211 conformation that mediates the interaction. Consequently the sequence recovery success will 212 largely depend on side-chain placement in a given backbone. The surface-centric design 213 approaches are compared to the standard FixBB Rosetta design protocol, using the ref2015 214 scoring function (60). In the presented benchmarks, the Rosetta FixBB design protocol serves as 215 baseline to assess the impact of surface-centric design on sequence recovery. 
216 In a first step, the target protein's interface is stripped off its native sequence by mutating all

217 interface residues to alanine. Second, the different design protocols were employed on the

218 interface positions with FixBB using the Rosetta energy function to select mutations while

219 RosettaSurf was guided by our surface similarity scoring term (Fig. 2A). RosettaSurf was

220 therefore provided with the native protein surface that we aimed to mimic. With this setup it is

221 possible to evaluate the sensitivity of the Surf score to recover native amino acids compared to

222 a known ground truth.

223 All different design protocols were employed for the bound and unbound states of the protein

224 complexes and results are reported by their category as mean sequence recovery rate (Fig. 2A).

225 Overall, sequence recovery rates were higher for surface-centric design pipelines while standard

226 Rosetta sequence design showed lower recovery rates regardless of the presence of the binder

227 (Fig. 2B). For all studied complexes, RosettaSurf and RosettaSurf-site outperformed FixBB by

228 36-39 percentage points in the unbound and 30-31 percentage points in the bound state. As

229 expected, sequence recovery was in general more successful in the presence of the binding

230 partner due to the reduced number of possible side-chains and rotameric conformations.

231 However, even in the presence of the binder, surface-centric design can be applied to improve

232 results. FixBB demonstrated improved performance for the antibody/antigen test set, however,

233 the surface-centric protocols still reached better results that were comparable to the other

234 categories. Worth noting is the $100 \%$ sequence recovery success of RosettaSurf-site for the D8

235 protein-vv138 antibody complex. The FixBB protocol performed slightly better for low-

236 complementary (55\%) than for high-complementarity complexes (48\%), while RosettaSurf and

237 RosettaSurf-site resulted in similar recovery rates of $\sim 85 \%$ in both cases with the notable

238 exception of RosettaSurf-site for low-complementarity complexes. Here, RosettaSurf-site was

239 able to recover $92 \%$ of the sequence and for one complex of that category, the Enterotoxin G -

240 T-cell receptor complex, even 100\%. 
241 Furthermore, we analyzed more closely outlier decoys, i.e. structures that scored high in surface

242 similarity but showed only little sequence recovery when designing with RosettaSurf. We set a

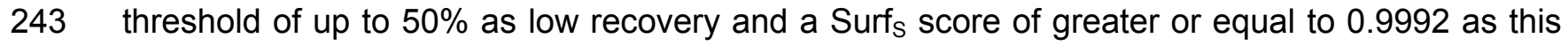

244 value marks the lower end of structures with high sequence recovery ( $>=70 \%)$, leading to one

245 candidate from the RSV epitope-scaffold-Motavizumab complex. When investigating which types

246 of amino acids were common failures, we identified general geometric resemblance as likely

247 reason. Several amino acids are frequently replaced by structurally similar residues, e.g.

248 isoleucine by valine and lysine by arginine. 
bioRxiv preprint doi: https://doi.org/10.1101/2021.06.16.448645: this version posted June 16, 2021. The copyright holder for this preprint (which was not certified by peer review) is the author/funder, who has granted bioRxiv a license to display the preprint in perpetuity. It is made available under aCC-BY 4.0 International license.

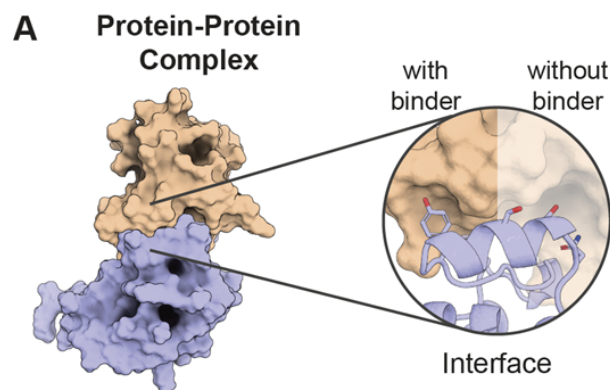

B

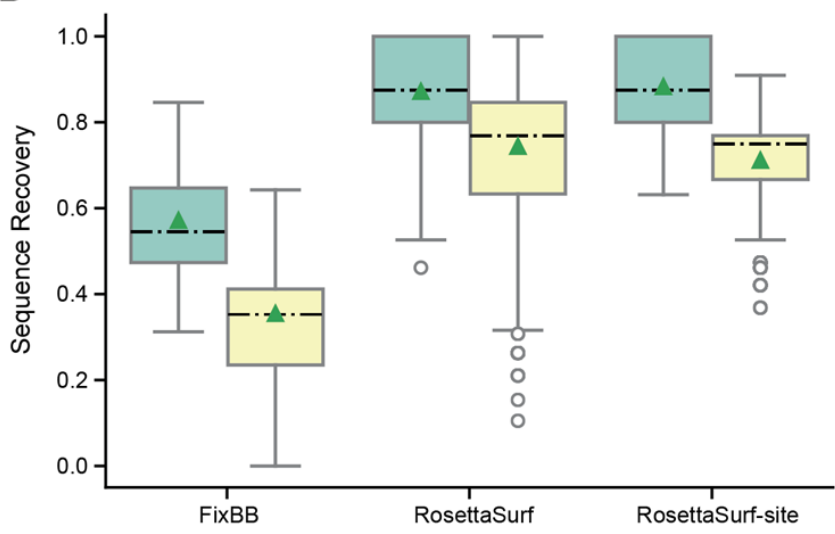

D

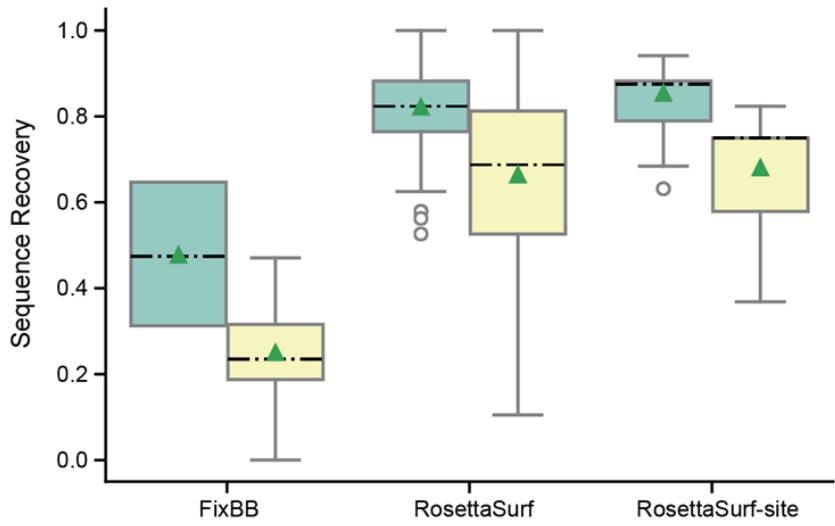

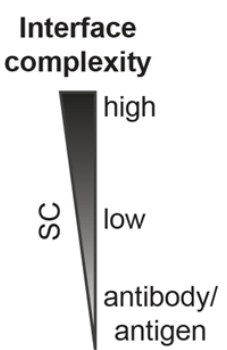
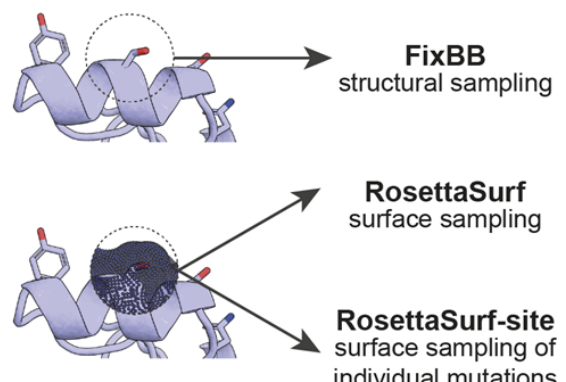

C

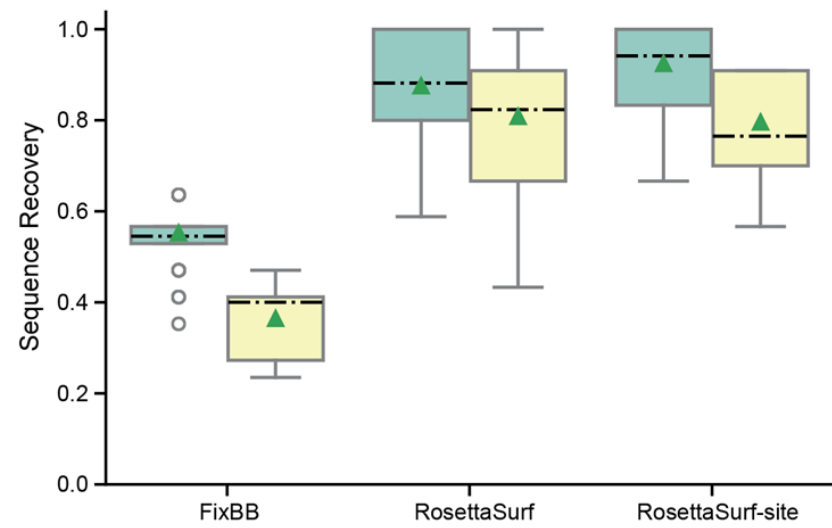

E

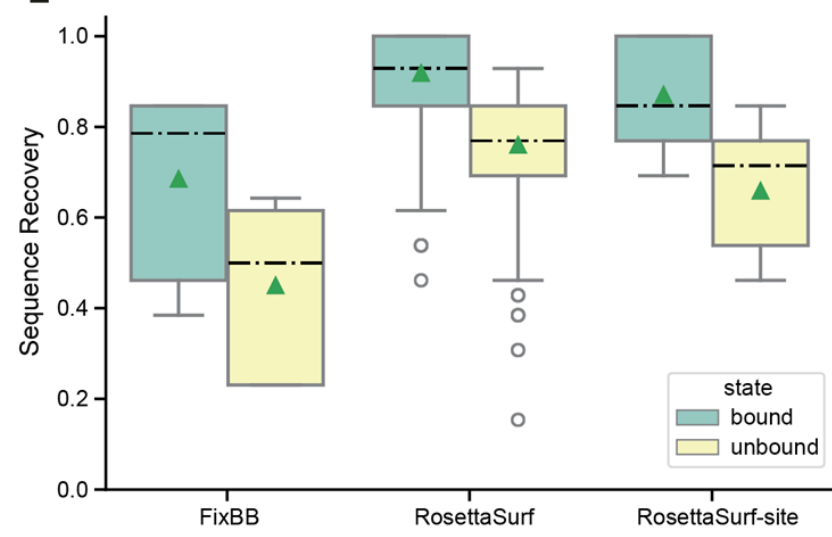

250 Figure 2. Sequence recovery of protein interfaces A) Sequence recovery benchmark pipeline. Sequences in the interfaces of protein-protein complexes are evaluated in the presence and absence of the binding partner. The tested complexes were grouped into interfaces with low and high shape complementarity, and antibody-antigen complexes. Surface-centric design (RosettaSurf and RosettaSurf-site) is compared to a standard structural protein design protocol

254 (FixBB). B) Interface sequence recovery of the complete dataset. C) Sequence recovery of low shape complementarity

255 interfaces. D) Sequence recovery of high shape complementarity interfaces. E) Sequence recovery of antigen-antibody

256 complexes. Dashed lines represent median and triangles represent mean recovery values.

257 As for sequence recovery without the binder, RosettaSurf and RosettaSurf-site performed better

258 for complexes of low shape complementarity with $81 \%$ and $80 \%$ recovery, respectively. However, 
sequence recovery for high-complementary and antibody/antigen complexes (66-76\%) was still substantially higher than for FixBB (25\% and $45 \%)$.

261 In addition, we investigated possible reasons for structures with low sequence recovery but high 262 surface similarity scores. We adjusted the selecting criteria to structures with $<=30 \%$ sequence 263 recovery and >= 0.9996 Surf $_{S}$ score, observing similar results as for the bound benchmark. Four 264 structures, all from the Colicin E9-IM9 complex fulfill these criteria. Again, mainly amino acids with 265 similar geometrical features were common mismatches between recovered and native amino 266 acids, e.g. phenylalanine, tyrosine, and histidine, aspartatic acid and asparagine, as well as valine 267 and threonine.

268 Overall, similar performance of RosettaSurf and RosettaSurf-site showed that performing the 269 combinatorial sampling of all possible rotamers simultaneously was not necessary. During the 270 RosettaSurf-site pipeline amino acids were sampled individually at each design position and only 271 a subset of the amino acids were used for combinatorial sampling based on the surface similarity 272 score, resulting in similar recovery outcomes.

273 Our results have thus implications for the design of functional proteins as the high success of

274 recovering natural protein interfaces may be promising for the design of proteins displaying 275 defined surface properties, as is the case for immunogen design.

\section{Computational SSM screening to improve protein binding}

278 A common problem in protein design is the optimization of PPIs to generate high affinity protein 279 binders. Experimental screening methods, e.g. site-saturation mutagenesis (SSM) and 280 combinatorial libraries, provide insights into mutations that improve binding interactions but are 281 time and resource-consuming experiments. We tested if RosettaSurf-site could be a fast and 282 efficient computational screening alternative to experimental based approaches. We studied the 283 optimization of de novo designed interleukin-2/15 antagonists that bind specifically to the IL-2R $\beta$ 
$284 \gamma_{c}$ receptor (61). The computationally designed interleukins were optimized for binding to the

285 interleukin receptor by performing SSM followed by combinatorial libraries based on the identified

286 mutations. The described study did not only report sequence information but included additional

287 structural characterization of the resulting design, thus allowing a fair comparison to our

288 computational method. This level of data completeness is rare in many other design endeavors

289 that have used this type of optimization strategy $(39,62)$.

290 We performed RosettaSurf-site similar to the approach described above (see "Protein Interface

291 Sequence Recovery"), however, with the selection criteria being shape complementarity of the

292 design and IL-2R $\beta \gamma_{c}$ interface rather than similarity as shape complementarity has been shown

293 to be a key feature of high affinity binding interactions (63). RosettaSurf-site allows exhaustive

294 computational sampling of amino acids similar to SSM experiments, screening all possible amino

295 acid substitutions at the interface of the interleukin design. Our study is based on the reported

296 crystal structure of the interleuking-2/15 design in complex with its cognate receptor (PDB: 6DG5).

297 We selected interface positions that were tested during SSM and later included in the

298 combinatorial library for our benchmark screening. Positions not interacting with the receptor, i.e.

299 not within a distance of $5 \AA$, were excluded from the selection, resulting in a total of five positions.

300 In our design protocol, the five selected residues were sampled with RosettaSurf-site and

301 mutations were evaluated based on the shape complementarity to the receptor surface. The

302 computational results were compared to the composition of the combinatorial library that was

303 constructed based on the preceding SSM screening. We analyzed whether RosettaSurf-site was

304 able to identify mutations that are present in the highest affinity binder as well as the performance

305 of the Rosetta energy function to retrieve similar mutations. After performing all possible mutations

306 with RosettaSurf-site, the four top-ranking amino acids for each position were selected (Fig. 3), in

307 line with the maximum number of amino acids included in the combinatorial library.

308 RosettaSurf-site recovered four out of the five mutations present in the best design, with residue

30939 being at the edge of the interface and contributing minimally to the protein-protein interaction, 
thus making it more challenging for this approach. Four additional residues included in the combinatorial library were recovered, that were observed to improve binding in the SSM but were not present in the highest affinity binder. In contrast, selecting mutations based on Rosetta energy alone recovers only a single mutation observed in the best binder and two residues present in the combinatorial library. These results show the potential of surface-based design of single point mutants as fast and promising approach to select candidates for combinatorial libraries to improve binding interactions without requiring preceding screening experiments like SSM.

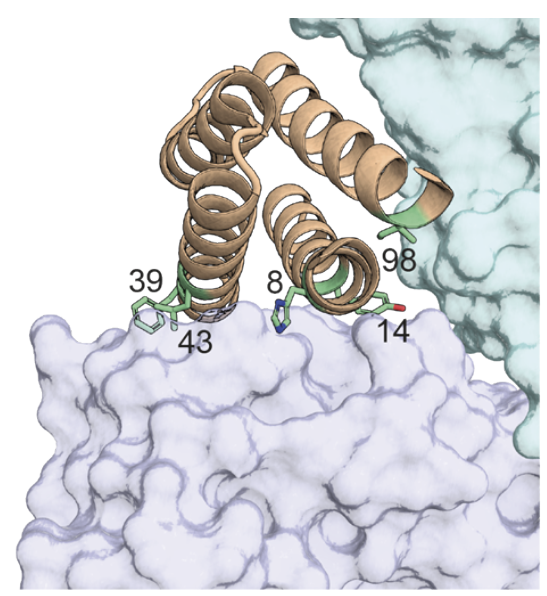

\begin{tabular}{c|c|c|c|c|c} 
Position & $\mathbf{8}$ & $\mathbf{1 4}$ & $\mathbf{3 9}$ & $\mathbf{4 3}$ & $\mathbf{9 8}$ \\
\hline Native & $\mathrm{L}$ & $\mathrm{L}$ & $\mathrm{S}$ & $\mathrm{V}$ & $\mathrm{I}$ \\
\hline \multirow{4}{*}{ sSM } & $\mathrm{Y}$ & $\mathrm{Y}$ & $\mathrm{F}$ & $\mathrm{F}$ & $\mathrm{N}$ \\
\cline { 2 - 6 } & $\mathrm{F}$ & $\mathrm{F}$ & $\mathrm{Y}$ & $\mathrm{L}$ & \\
\cline { 2 - 6 } & $\mathrm{H}$ & $\mathrm{H}$ & & & \\
\hline \multirow{4}{*}{$\begin{array}{c}\text { RosettaSurf } \\
\text { site }\end{array}$} & $\mathrm{F}$ & $\mathrm{Y}$ & $\mathrm{G}$ & $\mathrm{E}$ & $\mathrm{I}$ \\
\cline { 2 - 6 } & $\mathrm{Y}$ & $\mathrm{F}$ & & $\mathrm{Q}$ & $\mathrm{V}$ \\
\cline { 2 - 6 } & $\mathrm{N}$ & $\mathrm{L}$ & & $\mathrm{M}$ & $\mathrm{T}$ \\
\hline \multirow{4}{*}{ REU } & $\mathrm{H}$ & $\mathrm{R}$ & & $\mathrm{L}$ & $\mathrm{R}$ \\
\cline { 2 - 6 } & $\mathrm{R}$ & $\mathrm{A}$ & $\mathrm{K}$ & $\mathrm{N}$ & $\mathrm{I}$ \\
\cline { 2 - 6 } & $\mathrm{I}$ & $\mathrm{N}$ & $\mathrm{S}$ & $\mathrm{D}$ & $\mathrm{V}$ \\
\cline { 2 - 6 } & $\mathrm{D}$ & $\mathrm{E}$ & $\mathrm{N}$ & $\mathrm{Y}$ & $\mathrm{T}$ \\
\hline
\end{tabular}

Figure 3. Comparison of SSM data obtained for the designed interleukin-2/15 antagonists in comparison to

RosettaSurf-site predictions. The structure highlights the five selected positions of the interleukin design that were

computationally and experimentally sampled. Different sampling results of the experimental SSM, RosettaSurf-site with

SC, and Rosetta's energy function are reported in the table. Mutations resulting in the experimentally reported best

binding design are highlighted in dark green. RosettaSurf-site was able to recover four out of five key binding mutations

(dark green) while evaluating mutations with Rosetta's energy function could only retrieve one binding mutation at position 98. Additionally, RosettaSurf-site was able to recover four affinity improving mutations not present in the best binding design, whereas Rosetta's energy function could identify only two of these mutations (light green).

328 In recent years, protein design has shown promising results in the field of immunoengineering, allowing the computational design of epitope-focused immunogens that were shown to elicit functional antibody responses in mice and non-human primates $(34,36,37,42)$. To achieve an 
331 epitope-specific immune response, the epitope is transplanted from the viral antigen to an

332 unrelated small protein scaffold, presenting the antigenic site in isolation. We applied the

333 RosettaSurf protocol to the design of immunogens mimicking the antigenic site 0 , an epitope on

334 the F-glycoprotein of Respiratory Syncytial Virus (RSV) which consists of an irregular $\alpha$-helix and

335 a 10-residue long loop. Previous studies have shown that identifying scaffolds that can present

336 such complex structural motifs is challenging, and specifically for site 0 they are currently not

337 available in the known structure space. De novo design methods have been successfully used to

338 build scaffolds from scratch $(37,38)$, but the design process remains challenging and expertise in

339 de novo protein design is critical. The RosettaSurf approach allows to rescue protein scaffolds

340 that only present partial structural matches to the epitope structure motif.

341 As a demonstration of the use of RosettaSurf to transplant the epitope site into an unrelated

342 scaffold, a two-step strategy was employed: 1) side chain grafting of the $\alpha$-helical segment onto

343 a small, monomeric protein scaffold (Fig. 4A); 2) RosettaSurf design of the remaining antigenic

344 site, including the surface generated by the epitope loop and transition to the helical fragment

345 (Fig. 4A). The grafted helical segment serves as anchor point around which the surface can be

346 optimized to mimic the complete antigenic site.

347 We identified the NarX histidine kinase receptor (PDB: 3EZI) (59) as promising scaffold. The

348 small, monomeric protein can accommodate the epitope $\alpha$-helix (Ca RMSD: $0.4 \AA$ ) and offers a

349 sufficiently large surface area around the helix to be optimized with RosettaSurf. We generated

350760 designs using the RosettaSurf pipeline and the site 0 surface, as observed in complex with

351 site-specific antibody D25 (57), as a sequence optimization target. The best design was selected

352 based on the highest surface similarity score and was further optimized with six mutations

353 (Surf_03). First, five point mutations, which were not part of the interface, were introduced to

354 resolve clashes between native scaffold and epitope residues in the a-helix as well as steric

355 hindrance with residues introduced during surface-centric design. In addition, we mutated a polar 
residue in the binding pocket to a hydrophobic residue based on the sequence profile of the top 20 surface-designed decoys.

358 Two additional variants of the same protein scaffold were designed to test whether there were 359 advantages in using RosettaSurf. One design containing only the $\alpha$-helix of site 0 (RSV_helix) 360 and three point mutations that resolve clashes with the side chain grafted epitope helix, similar to 361 Surf_03. The second design was generated with FixBB (RSV_FixBB), starting with side-chain 362 grafting of the epitope $\alpha$-helix, and allowing to design the same amino acid positions as those of 363 Surf_03. The RosettaSurf design (Surf_03) reaches a surface similarity score of 0.45 compared 364 to the antigenic site in the native viral protein RSVF, while the native scaffold scores 0 . The helix365 grafted base-design scores 0.06 and the RSV_FixBB design 0.04 in surface resemblance.

366 To test experimentally our predictions, the designs were expressed and purified, and the binding 367 affinities were measured using a panel of monoclonal antibodies that engage the site 0 epitope 368 (Fig. 4B) $(37,57,64)$. In essence, this panel of monoclonal antibodies (mAbs) was used as conformational probes to assess the surface mimicry presented by the designs. We compared

370 the binding profiles of the three designs, a negative control (Surf_03 KO) and the WT scaffold 371 using surface plasmon resonance (Fig. S3). All three designs bound to the D25 and ADI19009 372 mAbs, indicating that these mAbs mostly rely on the helical segment of the epitope. Surf_03 was 373 the only design recognized by two additional antibodies (ADI14496 and ADI18900), indicating 374 that the higher surface mimicry achieved through RosettaSurf design improves the presentation 375 of the antigenic site and promotes binding of additional site-specific antibodies. The designed 376 immunogen demonstrates RosettSurf's capabilities to sculpt protein surfaces with a high degree 377 of accuracy which could be of use to introduce functional sites into protein scaffolds by optimizing 378 the molecular surface. 
bioRxiv preprint doi: https://doi.org/10.1101/2021.06.16.448645; this version posted June 16, 2021. The copyright holder for this preprint (which was not certified by peer review) is the author/funder, who has granted bioRxiv a license to display the preprint in perpetuity. It is made available under aCC-BY 4.0 International license.

A

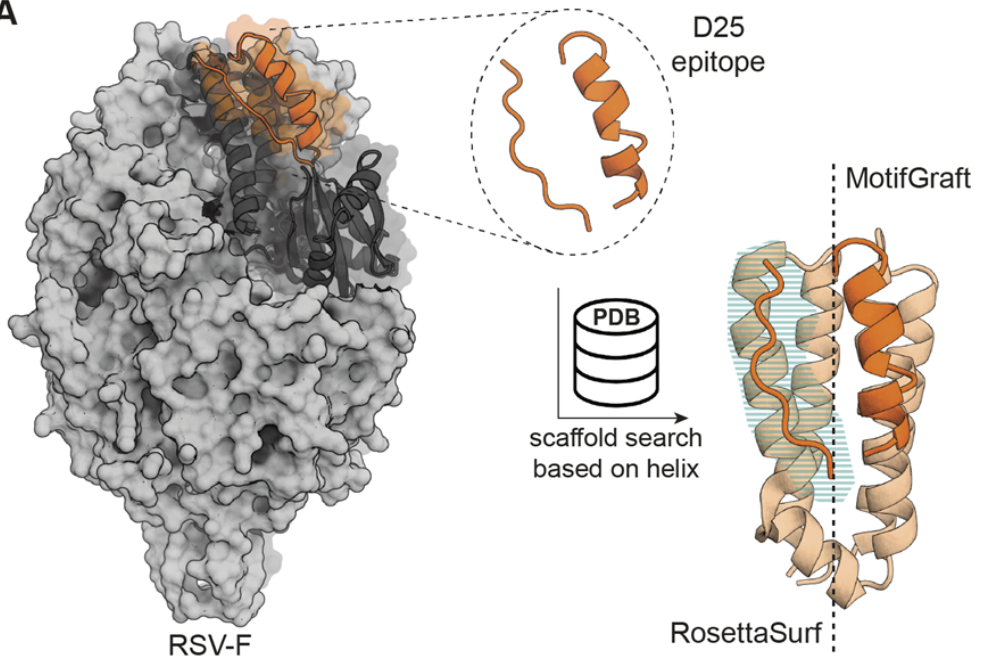

B

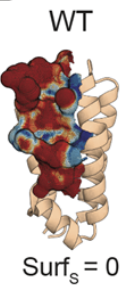

RSV_FixBB

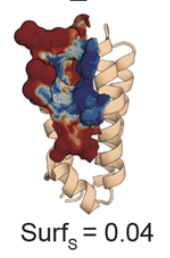

Surf 03

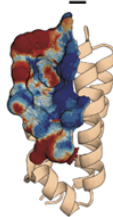

Surr $_{\mathrm{s}}=0.45$
C

ฉิ
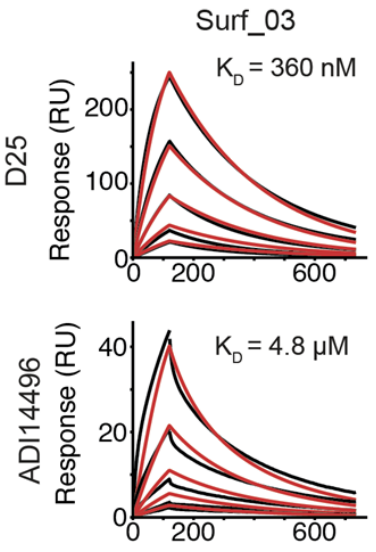

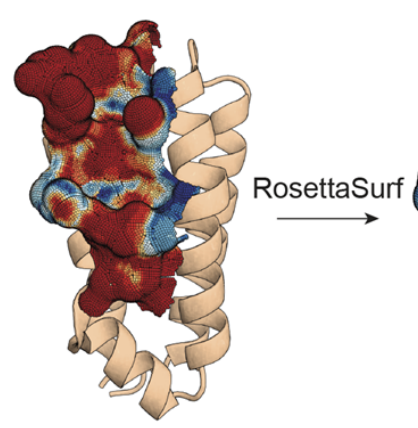

WT scaffold

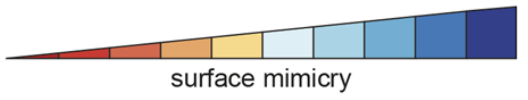

Figure 4. Surface centric design of a viral antigenic site present the in RSVF. A) Design process of site 0-mimicking protein scaffolds. Starting scaffolds are selected from the PDB based on structural alignments with the epitope helix.

The surface mimicking designs are generated by grafting the side chains of the helix segment of the epitope onto the scaffold and surface-centric design is employed to optimize the loop region. Before and after design of the surface compared to native site 0 . Blue areas indicate high similarity. B) Mimicry of surface geometry of WT scaffold, RSV_FixBB, and Surf_03 designs compared to native site 0. C) Representative SPR measurements of Surf_03 and RSV_FixBB against site 0-specific antibodies D25 and ADI14496. D) Binding profiles of Surf_03, a FixBB designed protein, and a helix-only design against a panel of site-specific antibodies with green indicating binding and red cells 
In this work we propose a surface-centric protein design approach and demonstrate its accuracy in several in silico benchmarking and experimental design tasks. The protocol can either be used

394 to optimize surface similarity or surface complementarity depending on the design task at hand.

395 Our ideas to leverage the surface geometrical and chemical features stem from observations that surfaces displaying similar patterns can have similar functional roles (e.g. in PPIs $(2,11,14,65))$. Technically, our framework is derived from earlier work by Lawrence and Coleman that introduced a fast and efficient way to evaluate shape complementarity as well as work by McCoy et al. that addressed electrostatic complementarity in interfaces. Based on some of these principles, we

400 further implemented a surface similarity score to evaluate both shape and chemistry of surfaces.

401 The resulting surface similarity score is implemented with a focus on surface-centric protein 402 design pipeline, allowing easy interaction and modification of the algorithm inside Rosetta design 403 protocols.

404 We showed the high accuracy of the surface score in recovering single amino acid identities by 405 their surface properties and show its benefit when used in conjunction with the Rosetta energy 406 score. Further, we highlight the use of surface similarity score inside a Monte Carlo sampling 407 approach and its performance in the sequence recovery of complete protein interfaces. The 408 incorporation of surface similarity clearly increases sequence recovery rates relative to other 409 scoring schemes.

410 This approach can be readily applied for the design of experimental combinatorial libraries to 411 reduce library sizes, as the high sequence recovery rates suggest that surface-centric design is 412 especially interesting to design novel proteins that mimic surface patches of other proteins, as it 413 is the case for the design of novel immunogens for vaccine development (36-38).

414 We demonstrated that surface-centric design could be used to generate targeted libraries to 415 optimize binding specificity of a previously reported IL-2 design (61). With the ranking of all 
416 mutations, we were able to recover amino acid variants that were also obtained by experimental

417 screening through a saturation mutagenesis library. This approach represents a straightforward

418 computational screening method to detect mutations that modulate specificity and affinity in

419 protein-protein interactions. Specifically, this strategy is a fast and accessible alternative to

420 experimental screening techniques.

421 Finally, we applied the surface-centric design protocol to engineer novel immunogens to present

422 the surface patch of an antigenic site present in the RSVF protein. We used a structurally complex

423 epitope (site 0 from RSVF), that consists of two structural segments, as an example for structurally

424 challenging sites that remain difficult for computational design approaches. The surface-centric

425 designs show a broader binding profile across a panel of monoclonal antibodies as compared to

426 other design approaches, suggesting that the surface presented is a closer mimic of the native

427 antigenic site.

428 Possible applications of surface-centric design range from the computational design of highly 429 specific protein-protein interactions, focusing on optimizing the surface complementarity both in

430 shape and chemistry. On the other hand, surface similarity enables the computational design of

431 proteins that may recapitulate precise surface features of target surfaces, which could then find

432 applications in immunogen design, where surface similarity allows the design of immunogens

433 mimicking an antigenic site of interest that could ultimately be used as probes for antibody

434 isolation or vaccine antigens.

435 Ultimately, we introduce a new conceptual approach in computational protein design where the

436 fine features of molecular surfaces are explicitly optimized. Through this route it will now be

437 possible to explore the tantalizing hypothesis of the existence of a "surface degeneracy code",

438 which in some cases may allow to represent similar surface patches using very distinct sets of

439 amino acids. Importantly, this capability could also enable the presentation of similar surface

440 patches in proteins with completely different backbone architectures which could again realize

441 many endeavors in functional protein design that are thus far out of reach. 


\section{Materials and Methods}

\section{RosettaSurf framework}

444 The RosettaSurf protocol operates at the solvent-excluded surface level of a protein structure, 445 and its core operation is the comparison between surfaces. To compare molecular surfaces, we 446 defined a score that quantifies the similarity between two surfaces considering both shape and 447 electrostatic features, and incorporated it within the Rosetta software package. The molecular 448 surface is generated from the three-dimensional atomic coordinates of a protein $(4,5)$ and stored 449 as a discrete point cloud (Fig. 5). Representation of the surface as a point cloud allows the

450 featurization of the points with geometrical and chemical descriptors (Fig. 5) and enables rapid 451 calculations of the surface similarity score. We refer to the mutable surface as target while the 452 reference surface used for comparisons is denoted as reference.

453 To describe the surface geometry, we developed a descriptor based on concepts introduced by

454 Lawrence and Colman (19) that quantifies shape relationships of two surfaces relying on normal 455 vector comparisons that are derived at each point of the surface. To evaluate surface shape 456 similarity, two protein structures are first aligned and the closest surface points between target 457 and reference are identified. Next, the normal vectors of the surface points are compared by first 458 computing their dot product to obtain the enclosed angle, followed by distance-based scaling that 459 penalizes points that are far apart (Fig. 5). Each comparison yields a shape similarity score for 460 the considered pair of points and the similarity of the entire surface results from computing the 461 mean of all pair-wise point comparisons. Since the identification of closest points depends on the 462 starting surface, the surface scores are not identical depending on which structure is the 463 considered the reference and the target and thus the scores are computed in both combinations. 464 A robust surface similarity score is obtained by considering the similarity of the target surface 465 compared to the reference surface and vice versa, effectively averaging the shape similarity to 466 correct for differences during the selection of closest points. 
467 Electrostatic similarity $\left(E_{S}\right)$ of two surfaces is derived from comparing their electrostatic potentials,

468 i.e. $E_{S}$ is assessed by computing the correlations of two electrostatic fields (Fig. 5). To quantify

469 surface electrostatics, the electrostatic potentials are computed over the continuous electrostatic

470 field and discrete charge values are assigned to every point of the surface using the APBS

471 software (46) (Fig. 5). The representation of the target and reference surface point clouds as

472 vectors allows fast computation of Pearson's and Spearman's rank correlations as described by

473 McCoy and colleagues (20). The resulting correlation coefficients capture the similarity of the

474 individual potential values as well as the overall trends in electrostatic similarity of the two surfaces

$475(18,20)$.

476 To accurately capture the relative contributions of both shape and electrostatics on the similarity 477 of molecular surfaces, we combined both scores into a single surface similarity score (Surf $\mathrm{S}_{\mathrm{S}}$ ).

478 Since we were interested in assessing similarity of surface sites that are well maintained and 479 relevant to functional protein design, we focused on interfaces of PPIs and performed logistic 480 regression on a dataset of 2,660 protein complexes to optimize the individual weights of the Surf

481 score for each component, shape and electrostatics. For each amino acid type (excluding 482 cysteines) a total of 140 complexes containing the respective amino acid in the interface region 483 were considered. All possible point mutations to non-native amino acids were generated and their 484 geometric and electrostatic similarity to the native surface measured, resulting in a dataset 485 containing shape and electrostatic properties for each mutation. Logistic regression was applied 486 to identify the optimal set of weights to combine shape and electrostatic features to optimize for 487 the highest recovery rates of the native amino acids.

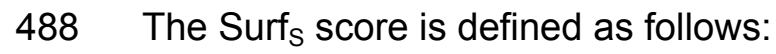

$$
\text { SurfS }=\frac{1}{1+\exp \left(13.79986756-14.64347448 \cdot S_{S}+13.78594078 \cdot E_{S}\right)}
$$

490 The final Surf s score combines both properties into a single score scaled from 0 to 1 , where 1 491 represents highly similar surfaces. We note that the developed surface similarity score can be 
bioRxiv preprint doi: https://doi.org/10.1101/2021.06.16.448645; this version posted June 16, 2021. The copyright holder for this preprint (which was not certified by peer review) is the author/funder, who has granted bioRxiv a license to display the preprint in perpetuity. It is made available under aCC-BY 4.0 International license.
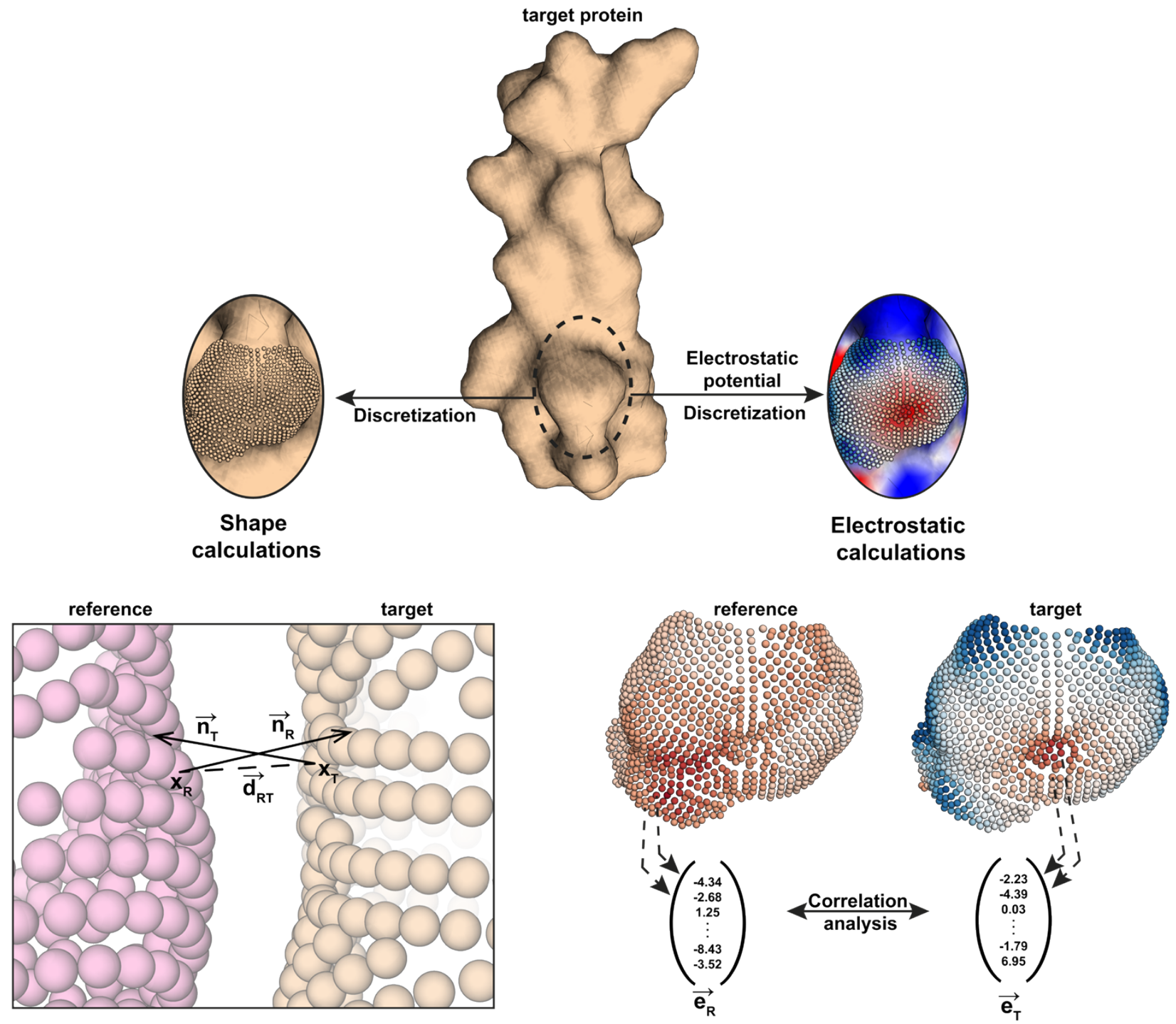

496 Figure 6. Computation of the surface similarity score (Surf $f_{\mathrm{S}}$ ). Protein surface is converted into a point cloud where

each point is used to compute shape and electrostatic features. To compute the Surf score, all individual points of the 


\section{Surface-centric protein design protocol}

502 We developed the RosettaSurf design protocol, as part of the RosettaScript software environment

503 (47), which utilizes the described surface scoring function during the sequence sampling stage of

504 the design process to bias the selection of amino acids and rotamers towards a desired surface

505 configuration in terms of geometric and electrostatic properties (Fig. 2A). Thereby, it is possible

506 to design proteins for function without relying on protein grafting but by optimizing the molecular

507 surface, for instance, to mimic a given active site.

508 With the RosettaSurf design protocol we explicitly optimize molecular surface features during the

509 protein design process. In practice, RosettaSurf performs sequence design in a way where the

510 mutable surface of the design scaffold (target) is optimized to closely match the surface features

511 of a reference protein (reference) (Fig. S1). To efficiently explore the sequence space during the

512 design process, Monte Carlo simulated annealing guides the optimization of rotamers, where

513 substitutions of residues are scored based on the resulting surface and accepted if they pass the

514 Monte Carlo criterion that is implemented as the Surf score. $^{2}$

515 To reduce computational time spent on rotamer sampling in a combinatorial fashion on the overall

516 surface, we implemented a single amino acid scanning surface-centric protein design approach

517 (RosettaSurf-site). This protocol samples amino acids individually at each position of the design

518 surface, selects the top three rotamers according to the surface and samples those

519 combinatorially with other designable positions, reducing the combinatorial possibilities.

\section{Benchmark datasets}

522 The dataset of the single amino acid recovery benchmark consists of 1,900 protein complexes,

523 with 100 complexes for each amino acid identity except cysteine. We divided each of these protein

524 complexes into target proteins, where the interface can be mutated, and binders, the proteins that

525 serve as context for the target. The interface of the target protein is defined as residues that are 
526 within $7 \AA C_{\beta}$-distance of the binder and have the $C_{\beta}$-atom pointing towards the binder. This

527 selection ensures that the amino acid side chains are part of the interface and the contribution of

528 the residue to the binding interaction is not solely due to backbone interactions. The full target

529 interface is converted to alanine, effectively removing any side chain memory of the native

530 structure that would restrict the placement of new rotamers and introduce biases towards the

531 native sequence. Cysteine residues are ignored as they can form chemical linkages in the form

532 of disulfide bonds, thus generating a surface that is not attributable to an individual residue.

534 To test the performance of the surface scoring function we sought to perform a benchmark to evaluate sequence recovery at the interfaces of protein-protein interactions with varying shape complementarity. We assembled a diverse dataset consisting of nine protein complexes capturing

537 different aspects of PPIs and grouped them into three different categories: 1) low-shape 538 complementary interactions which include Enterotoxin G - T-cell receptor complex (PDB: 3MC0)

539 (48), Ribonuclease A in complex with its inhibitor (PDB: 1DFJ) (49) and domain 2 of VEGFR1 in complex with PIGF (PDB: 1RV6) (50); 2) High-shape complementarity interactions which include

541 the complex between Colicin E9 and IM9 (PDB: 1EMV) (51), Bovine beta-trypsin in complex with

542 CMTI-I (PDB: 1PPE) (52) and PD-L1 in complex with a nanobody (PDB: 5JDS) (53); 3) antigen-

543 antibody interactions which include HIV-gp120 in complex with CD4-binding site antibody b13

544 (PDB: 3IDX) (54), RSV epitope-scaffold in complex with Motavizumab (PDB: 4JLR) (34) and the

545 Vaccinia virus D8 protein in complex with the antibody vv138 (PDB: 6B9J) (55). The shape

546 complementarity of the complexes in the first two categories was assessed by the Rosetta shape-

547 complementarity filter. Protein complexes with a shape complementarity score less than 0.65

548 were classified as low-complementarity. During the benchmark analysis we distinguish between

549 bound and unbound proteins, where unbound proteins are obtained by removing the protein

550 binder from the holo-crystal structure.

551 Data analysis was performed with the help of the rstoolbox Python library (56). 


\section{Computational design of proteins mimicking the antigenic site 0 in RSV}

554 The structure of antigenic site 0 was extracted from the crystal structure of prefusion stabilized

555 RSVF in complex with antibody D25 (PDB: 4JHW) (57). The D25 epitope consists of an irregular 556 a-helix (residues 196-209) and a 10-residue loop (residues 61-70). To identify putative scaffolds,

557 we performed a structural search based on the irregular $\alpha$-helix against 55,574 monomeric, helix-

558 containing crystal structures from the Protein Data Bank (PDB, from September 2015) using

559 Rosetta's MotifGraft algorithm (58). Matches were filtered at a backbone RMSD threshold below

$560 \quad 0.55 \AA$ and less than ten atomic clashes at the interface, resulting in 13 scaffold candidates. After

561 visual inspection, we selected the NarX histidine kinase receptor (PDB: 3EZI) (59) as scaffold, a

562 107-residue long, monomeric protein that aligned to the epitope helix with a $\mathrm{C}_{\alpha}$ RMSD of $0.4 \AA$

563 and provided additional surface area to mimic the entire antigenic site.

564 In a first step, we transplanted the side chains of the epitope helix contacting the D25 antibody

565 onto the selected scaffold using MotifGraft, followed by the introduction of three mutations on the

566 protein scaffold that were not part of the interface to resolve steric clashes with the transferred

567 side chains, resulting in the design RSV_helix. Subsequently, we performed surface-centric

568 design on 19 residues using the RosettaSurf pipeline to increase surface mimicry of the site 0

569 around the epitope helix, resulting in 760 design decoys. Surface-centric design was performed

570 in the presence of the D25 antibody and site 0 of RSVF served as a reference to which our

571 designed surface was optimized. We selected the decoy with the highest surface mimicry score,

572 named surf_01, and optimized the design in additional steps. To allow accurate display of the

573 designed surface, we introduced two point mutants in the scaffold adjacent to the optimized

574 surface patch to avoid steric hindrance (surf_02). Lastly, after comparing the similarity of surf_02

575 and the native antigenic site, we identified a lysine residue at position 18 in our design with

576 suboptimal mimicry. Evaluating the sequence profile of the 20 best scoring decoys sorted by 
577 surface mimicry revealed a strong preference for leucine at this position and the residue was

578 incorporated into a new design (Surf_03). Finally, we designed a version of RSV_helix that used

579 Rosetta's fixed-backbone design (FixBB) to serve as comparison to our surface-optimized

580 designs. We designed 1'000 decoys in the presence of D25 antibody, allowing mutations to occur

581 at the same 19 residues as was the case for RosettaSurf design. All designs converged to an

582 identical sequence which was selected as design RSV_FixBB. Based on Surf_03 we designed a

583 knockout mutant (Surf_03_KO) with a N74Y mutation in the epitope helix.

Protein expression and purification

Designs

587 Genes for all designs were purchased as DNA fragments from Twist Bioscience, and cloned into 588 pET11 vectors, containing a N-terminal MBP-tag and His-tag as well as a TEV cleavage site, for 589 bacterial expression. Plasmids were transformed into E. coli BL21 (DE3 pLysS) (Merck, \#69451590 3) and grown overnight in LB media at $37^{\circ} \mathrm{C}$. Pre-cultures were diluted $1: 50$ and inoculated to an $591 \mathrm{OD}_{600}$ of 0.6 in terrific broth (Condalab, \#PRO1246.05) at $37^{\circ} \mathrm{C}$ and expression was induced by 592 the addition of $1 \mathrm{mM}$ isopropyl-ß-D-thiogalactoside (IPTG). Cultures were harvested after 18-20 593 hours at $20^{\circ} \mathrm{C}$. Pellets were resuspended in lysis buffer $(50 \mathrm{mM}$ Tris, pH 7.5, $500 \mathrm{mM} \mathrm{NaCl}, 5 \%$ 594 Glycerol, $1 \mathrm{mg} / \mathrm{ml}$ lysozyme, $1 \mathrm{mM} \mathrm{PMSF}$, and $1 \mu \mathrm{g} / \mathrm{ml}$ DNase) and sonicated on ice for a total of 59512 minutes, in intervals of 15 s sonication followed by $45 \mathrm{~s}$ pause. The lysates were cleared by 596 centrifugation (48'384 g, $20 \mathrm{~min}$ ) and purified using a His-Trap FF column on an Äkta pure system 597 (GE Healthcare), followed by size exclusion on a HiLoad 16/600 Superdex 75 column (GE 598 Healthcare) in phosphate-buffered saline (PBS). Protein concentrations were determined by 599 measuring the absorbance at $280 \mathrm{~nm}$ on a Nanodrop (Thermo Scientific). The designed proteins 600 were concentrated by centrifugation (Millipore, \#UFC900324) to $1 \mathrm{mg} / \mathrm{ml}$, snap frozen in liquid 601 nitrogen, and stored at $-80^{\circ} \mathrm{C}$. 
603 Antibody variable fragments (Fabs)

604 For Fab expression, heavy and light chain DNA sequences were purchased from Twist 605 Biosciences and cloned separately into the pHLSec mammalian expression vector (Addgene, 606 \#99845) using Agel and Xhol restriction sites. Expression plasmids were premixed in a 1:1 607 stoichiometric ratio, co-transfected into HEK293-F cells, and cultured in FreeStyle medium 608 (Gibco, \#12338018). Supernatants were harvested after 1 week by centrifugation and purified 609 using a kappa-select column (GE Healthcare). Elution of bound proteins was conducted using 0.1

$610 \mathrm{M}$ glycine buffer ( $\mathrm{pH} 2.7)$, and eluates were immediately neutralized by the addition of $1 \mathrm{M}$ Tris 611 ethylamine ( $\mathrm{pH}$ 9), followed by buffer exchange to PBS ( $\mathrm{pH} 7.4)$.

613 Binding affinity determination by surface plasmon resonance (SPR)

614 SPR measurements were performed on a Biacore 8K (GE Healthcare) with HBS-EP+ as running 615 buffer (10 mM HEPES pH 7.4, $150 \mathrm{mM} \mathrm{NaCl,} 3 \mathrm{mM}$ EDTA, 0.005\% v/v Surfactant P20, GE 616 Healthcare) at room temperature. Approximately 700 response units (RU) of Fabs were 617 immobilized on a CM5 sensor chip (GE Healthcare) via amine coupling, and designed monomeric 618 proteins were injected as analyte in two-fold serial dilutions. The flow rate was $30 \mu \mathrm{l} / \mathrm{min}$ with 120 619 s of contact time followed by 400 s dissociation time. After each injection, surface was regenerated 620 using $0.1 \mathrm{M}$ glycine at $\mathrm{pH}$ 3.5. Data were fitted using 1:1 Langmuir binding model within the 621 Biacore 8K analysis software (GE Healthcare \#29310604).

\section{Author contributions}

624 A.S. and B.E.C. conceived the work and designed the experiments. A.S. implemented the 625 described protocol with input from J.B. and performed computational design simulations and 
626 benchmark analysis. S.R. expressed and purified the reported proteins and performed SPR

627 assays. A.S., M.D., and A.L. developed the surface similarity score. A.S. and B.E.C wrote the 628 paper. All authors commented on the manuscript.

629

631 We would like to thank Pablo Gainza, Fabian Sesterhenn, Che Yang, and Sarah Wehrle for helpful

632 discussions and support regarding in vitro experiments. We thank Sailan Shui, Freyr Sverrisson,

633 and Arne Schneuing for comments on the manuscript. We also thank Luki Goldschmidt and Brian

634 Coventry for implementing the shape and electrostatic complementarity evaluation within Rosetta,

635 respectively. Additionally, we would like to acknowledge the high-performance computing facility

636 (SCITAS) for their technical support. We would also like to acknowledge the Swiss National

637 Supercomputing Centre (CSCS) for their support in computing time. This work was supported by

638 the European Research Council (starting grant no. 716058), the Swiss National Science

639 Foundation (grant no. 310030_188744), the NCCR Molecular Systems Engineering and the

640 NCCR Chemical Biology. Andreas Loukas was supported by the Swiss National Science

641 Foundation project “Deep Learning for Graph-Structured Data” (grant number PZO0P2 179981).

642 Jaume Bonet was funded by the EPFL Fellows postdoctoral fellowship. The funders had no role

643 in study design, data collection and analysis, decision to publish, or preparation of the manuscript.

\section{Data availability statement}

646 All data and scripts necessary to recreate the analysis and design simulations described in this 647 work are available at https://github.com/LPDI-EPFL/RosettaSurf. 


\section{References}

650

651

652

653

654

655

656

657

658

659

660

661

662

663

664

665

666

667

668

669

670

671

672

673

674

675

676

677

678

679

680

681

682

683

684

1. Kihara D, Sael L, Chikhi R, Esquivel-Rodriguez J. Molecular surface representation using 3D Zernike descriptors for protein shape comparison and docking. Current Protein and Peptide Science. 2011;12(6):520-30.

2. Shulman-Peleg A, Nussinov R, Wolfson HJ. Recognition of functional sites in protein structures. Journal of molecular biology. 2004;339(3):607-33.

3. Lee B, Richards FM. The interpretation of protein structures: estimation of static accessibility. Journal of molecular biology. 1971;55(3):379-IN4.

4. Connolly ML. Analytical molecular surface calculation. Journal of applied crystallography. 1983;16(5):548-58.

5. Connolly ML. Solvent-accessible surfaces of proteins and nucleic acids. Science. 1983;221(4612):709-13.

6. Richards FM. Areas, Volumes, Packing, and Protein Structure. Annu Rev Biophys Bioeng. 1977 Jun;6(1):151-76.

7. Ryu J, Park R, Kim D-S. Molecular surfaces on proteins via beta shapes. Computer-Aided Design. 2007;39(12):1042-57.

8. Greer J, Bush BL. Macromolecular shape and surface maps by solvent exclusion. Proceedings of the National Academy of Sciences. 1978 Jan 1;75(1):303-7.

9. Schmitt S, Kuhn D, Klebe G. A New Method to Detect Related Function Among Proteins Independent of Sequence and Fold Homology. Journal of Molecular Biology. 2002 Oct 18;323(2):387-406.

10. Kinoshita $\mathrm{K}, \mathrm{Nakamura} \mathrm{H}$. Identification of protein biochemical functions by similarity search using the molecular surface database eF-site. Protein Science. 2003;12(8):1589-95.

11. Yin S, Proctor EA, Lugovskoy AA, Dokholyan NV. Fast screening of protein surfaces using geometric invariant fingerprints. Proceedings of the National Academy of Sciences. 2009;106(39):16622-6.

12. Daberdaku S, Ferrari C. Fast Computation of High-resolution Solvent Excluded Protein Surface with OpenMP. In: 2018 International Conference on High Performance Computing Simulation (HPCS). 2018. p. 789-96.

13. Natarajan V, Koehl P, Wang Y, Hamann B. Visual analysis of biomolecular surfaces. In: Visualization in Medicine and Life Sciences. Springer; 2008. p. 237-55.

14. Gainza P, Sverrisson F, Monti F, Rodola E, Boscaini D, Bronstein M, et al. Deciphering interaction fingerprints from protein molecular surfaces using geometric deep learning. Nature Methods. 2020;17(2):184-92.

15. Sverrisson F, Feydy J, Correia BE, Bronstein MM. Fast end-to-end learning on protein surfaces. bioRxiv. 2020 Dec 29;2020.12.28.424589. 
685

686

687

688

689

690

691

692

693

694

695

696

697

698

699

700

701

702

703

704

705

706

707

708

709

710

711

712

713

714

715

716

717

718

719

720

16. Morris RJ, Najmanovich RJ, Kahraman A, Thornton JM. Real spherical harmonic expansion coefficients as 3D shape descriptors for protein binding pocket and ligand comparisons. Bioinformatics. 2005;21(10):2347-55.

17. Sharp KA, Honig B. Electrostatic interactions in macromolecules: theory and applications. Annual review of biophysics and biophysical chemistry. 1990;19(1):301-32.

18. Chau P-L, Dean PM. Electrostatic complementarity between proteins and ligands. 1. Charge disposition, dielectric and interface effects. J Computer-Aided Mol Des. 1994 Oct 1;8(5):513-25.

19. Lawrence MC, Colman PM. Shape complementarity at protein/protein interfaces. Elsevier; 1993.

20. McCoy AJ, Epa VC, Colman PM. Electrostatic complementarity at protein/protein interfaces. Journal of molecular biology. 1997;268(2):570-84.

21. Gabb HA, Jackson RM, Sternberg MJ. Modelling protein docking using shape complementarity, electrostatics and biochemical information. Journal of molecular biology. 1997;272(1):106-20.

22. Yan $Y$, Huang S-Y. Pushing the accuracy limit of shape complementarity for protein-protein docking. BMC bioinformatics. 2019;20(25):1-10.

23. Chen R, Li L, Weng Z. ZDOCK: an initial-stage protein-docking algorithm. Proteins: Structure, Function, and Bioinformatics. 2003;52(1):80-7.

24. Helmer-Citterich M, Tramontano A. PUZZLE: a new method for automated protein docking based on surface shape complementarity. Journal of molecular biology. 1994;235(3):102131.

25. Duhovny D, Nussinov R, Wolfson HJ. Efficient unbound docking of rigid molecules. In: International workshop on algorithms in bioinformatics. Springer; 2002. p. 185-200.

26. Zhu $\mathrm{X}$, Xiong $\mathrm{Y}$, Kihara D. Large-scale binding ligand prediction by improved patch-based method Patch-Surfer2. 0. Bioinformatics. 2014;31(5):707-13.

27. Venkatraman V, Sael L, Kihara D. Potential for protein surface shape analysis using spherical harmonics and 3D Zernike descriptors. Cell biochemistry and biophysics. 2009;54(1-3):23-32.

28. Huang P-S, Oberdorfer G, Xu C, Pei XY, Nannenga BL, Rogers JM, et al. High thermodynamic stability of parametrically designed helical bundles. science. 2014;346(6208):481-5.

29. Joh NH, Wang T, Bhate MP, Acharya R, Wu Y, Grabe M, et al. De novo design of a transmembrane Zn2+-transporting four-helix bundle. Science. 2014;346(6216):1520-4.

30. Kuhlman B, Dantas G, Ireton GC, Varani G, Stoddard BL, Baker D. Design of a novel globular protein fold with atomic-level accuracy. science. 2003;302(5649):1364-8. 
721

722

723

724

725

726

727

728

729

730

731

732

733

734

735

736

737

738

739

740

741

742

743

744

745

746

747

748

749

750

751

752

753

754

755

756

757

31. Siegel JB, Zanghellini A, Lovick HM, Kiss G, Lambert AR, Clair JLS, et al. Computational design of an enzyme catalyst for a stereoselective bimolecular Diels-Alder reaction. Science. 2010;329(5989):309-13.

32. Fleishman SJ, Whitehead TA, Ekiert DC, Dreyfus C, Corn JE, Strauch E-M, et al. Computational design of proteins targeting the conserved stem region of influenza hemagglutinin. Science. 2011;332(6031):816-21.

33. Tinberg CE, Khare SD, Dou J, Doyle L, Nelson JW, Schena A, et al. Computational design of ligand-binding proteins with high affinity and selectivity. Nature. 2013;501(7466):212-6.

34. Correia BE, Bates JT, Loomis RJ, Baneyx G, Carrico C, Jardine JG, et al. Proof of principle for epitope-focused vaccine design. Nature. 2014;507(7491):201-6.

35. Bonet J, Wehrle S, Schriever K, Yang C, Billet A, Sesterhenn F, et al. Rosetta FunFolDesA general framework for the computational design of functional proteins. PLoS computational biology. 2018;14(11):e1006623.

36. Sesterhenn F, Galloux M, Vollers SS, Csepregi L, Yang C, Descamps D, et al. Boosting subdominant neutralizing antibody responses with a computationally designed epitopefocused immunogen. PLoS biology. 2019;17(2):e3000164.

37. Sesterhenn F, Yang C, Bonet J, Cramer JT, Wen X, Wang Y, et al. De novo protein design enables the precise induction of RSV-neutralizing antibodies. Science. 2020;368(6492).

38. Yang C, Sesterhenn F, Bonet J, van Aalen EA, Scheller L, Abriata LA, et al. Bottom-up de novo design of functional proteins with complex structural features. Nature Chemical Biology. 2021;1-9.

39. Procko E, Berguig GY, Shen BW, Song Y, Frayo S, Convertine AJ, et al. A computationally designed inhibitor of an Epstein-Barr viral Bcl-2 protein induces apoptosis in infected cells. Cell. 2014;157(7):1644-56.

40. Chevalier A, Silva D-A, Rocklin GJ, Hicks DR, Vergara R, Murapa P, et al. Massively parallel de novo protein design for targeted therapeutics. Nature. 2017;550(7674):74-9.

41. Janda CY, Dang LT, You C, Chang J, de Lau W, Zhong ZA, et al. Surrogate Wnt agonists that phenocopy canonical Wnt and $\beta$-catenin signalling. Nature. 2017;545(7653):234-7.

42. Correia BE, Ban Y-EA, Holmes MA, Xu H, Ellingson K, Kraft Z, et al. Computational Design of Epitope-Scaffolds Allows Induction of Antibodies Specific for a Poorly Immunogenic HIV Vaccine Epitope. Structure. 2010 Sep 8;18(9):1116-26.

43. McLellan JS, Correia BE, Chen M, Yang Y, Graham BS, Schief WR, et al. Design and Characterization of Epitope-Scaffold Immunogens That Present the Motavizumab Epitope from Respiratory Syncytial Virus. Journal of Molecular Biology. 2011 Jun 24;409(5):853-66.

44. Azoitei ML, Correia BE, Ban Y-EA, Carrico C, Kalyuzhniy O, Chen L, et al. ComputationGuided Backbone Grafting of a Discontinuous Motif onto a Protein Scaffold. Science. 2011 Oct 21;334(6054):373-6. 
758

759

760

761

762

763

764

765

766

767

768

769

770

771

772

773

774

775

776

777

778

779

780

781

782

783

784

785

786

787

788

789

790

791

792

793

794

795

45. Leaver-Fay A, Tyka M, Lewis SM, Lange OF, Thompson J, Jacak R, et al. ROSETTA3: an object-oriented software suite for the simulation and design of macromolecules. Methods in enzymology. 2011;487:545-74.

46. Jurrus E, Engel D, Star K, Monson K, Brandi J, Felberg LE, et al. Improvements to the APBS biomolecular solvation software suite. Protein Science. 2018;27(1):112-28.

47. Fleishman SJ, Leaver-Fay A, Corn JE, Strauch E-M, Khare SD, Koga N, et al. RosettaScripts: a scripting language interface to the Rosetta macromolecular modeling suite. PloS one. 2011;6(6):e20161.

48. Fernández MM, Cho S, De Marzi MC, Kerzic MC, Robinson H, Mariuzza RA, et al. Crystal structure of staphylococcal enterotoxin G (SEG) in complex with a mouse T-cell receptor $\beta$ chain. Journal of Biological Chemistry. 2011;286(2):1189-95.

49. Kobe B, Deisenhofer J. A structural basis of the interactions between leucine-rich repeats and protein ligands. Nature. 1995;374(6518):183-6.

50. Christinger HW, Fuh G, de Vos AM, Wiesmann C. The crystal structure of placental growth factor in complex with domain 2 of vascular endothelial growth factor receptor-1. Journal of Biological Chemistry. 2004;279(11):10382-8.

51. Kühlmann UC, Pommer AJ, Moore GR, James R, Kleanthous C. Specificity in protein-protein interactions: the structural basis for dual recognition in endonuclease colicin-immunity protein complexes. Journal of molecular biology. 2000;301(5):1163-78.

52. Bode W, Greyling HJ, Huber R, Otlewski J, Wilusz T. The refined 2.0 LAA X-ray crystal structure of the complex formed between bovine $\beta$-trypsin and CMTI-I, a trypsin inhibitor from squash seeds (Cucurbita maxima) Topological similarity of the squash seed inhibitors with the carboxypeptidase A inhibitor from potatoes. FEBS letters. 1989;242(2):285-92.

53. Zhang $F$, Wei $H$, Wang $X$, Bai $Y$, Wang $P$, Wu J, et al. Structural basis of a novel PD-L1 nanobody for immune checkpoint blockade. Cell discovery. 2017;3(1):1-12.

54. Chen L, Do Kwon Y, Zhou T, Wu X, O'Dell S, Cavacini L, et al. Structural basis of immune evasion at the site of CD4 attachment on HIV-1 gp120. Science. 2009;326(5956):1123-7.

55. Matho MH, Schlossman A, Gilchuk IM, Miller G, Mikulski Z, Hupfer M, et al. Structurefunction characterization of three human antibodies targeting the vaccinia virus adhesion molecule D8. Journal of Biological Chemistry. 2018;293(1):390-401.

56. Bonet J, Harteveld Z, Sesterhenn F, Scheck A, Correia BE. rstoolbox - a Python library for large-scale analysis of computational protein design data and structural bioinformatics. BMC Bioinformatics. 2019 Dec;20(1):240.

57. McLellan JS, Chen M, Leung S, Graepel KW, Du X, Yang Y, et al. Structure of RSV Fusion Glycoprotein Trimer Bound to a Prefusion-Specific Neutralizing Antibody. Science. 2013 May 31;340(6136):1113-7.

58. Silva D-A, Correia BE, Procko E. Motif-Driven Design of Protein-Protein Interfaces. In: Stoddard BL, editor. Computational Design of Ligand Binding Proteins [Internet]. New York, 
NY: Springer New York; 2016 [cited 2021 Feb 22]. p. 285-304. (Methods in Molecular Biology; vol. 1414). Available from: http://link.springer.com/10.1007/978-1-4939-3569-7_17

59. Cheung J, Hendrickson WA. Structural analysis of ligand stimulation of the histidine kinase NarX. Structure. 2009;17(2):190-201.

60. Alford RF, Leaver-Fay A, Jeliazkov JR, O'Meara MJ, DiMaio FP, Park H, et al. The Rosetta all-atom energy function for macromolecular modeling and design. Journal of chemical theory and computation. 2017;13(6):3031-48.

61. Silva D-A, Yu S, Ulge UY, Spangler JB, Jude KM, Labão-Almeida C, et al. De novo design of potent and selective mimics of IL-2 and IL-15. Nature. 2019;565(7738):186-91.

62. Whitehead TA, Chevalier A, Song Y, Dreyfus C, Fleishman SJ, De Mattos C, et al. Optimization of affinity, specificity and function of designed influenza inhibitors using deep sequencing. Nat Biotechnol. 2012 Jun;30(6):543-8.

63. Yan Y, Huang S-Y. Protein-protein docking with improved shape complementarity. In: International Conference on Intelligent Computing. Springer; 2018. p. 600-5.

64. Gilman MSA, Castellanos CA, Chen M, Ngwuta JO, Goodwin E, Moin SM, et al. Rapid profiling of RSV antibody repertoires from the memory B cells of naturally infected adult donors. Science Immunology [Internet]. 2016 Dec 9 [cited 2021 May 5];1(6). Available from: https://immunology.sciencemag.org/content/1/6/eaaj1879

65. Keskin O, Nussinov R. Similar Binding Sites and Different Partners: Implications to Shared Proteins in Cellular Pathways. Structure. 2007 Mar 1;15(3):341-54. 


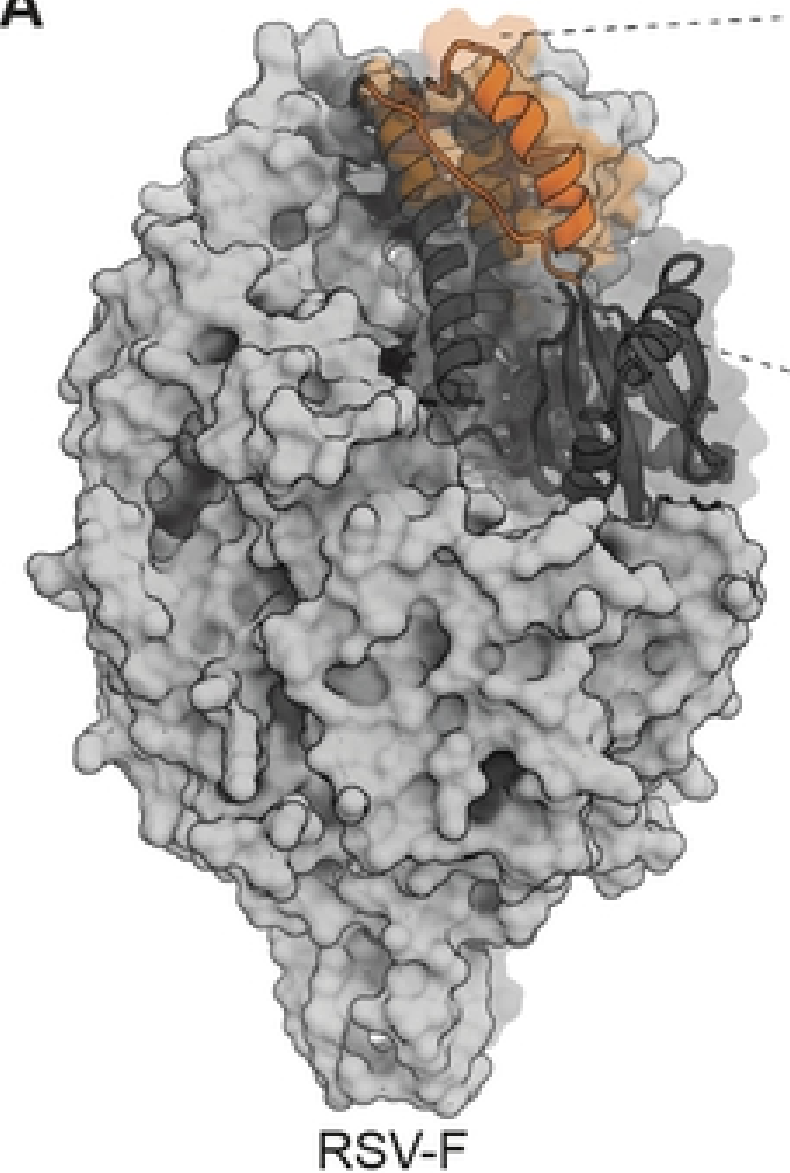

B

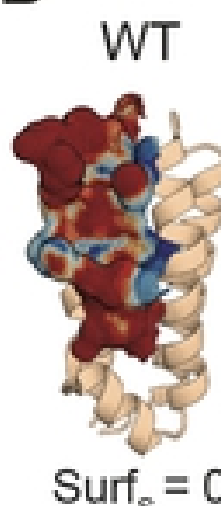

RSV_FixBB

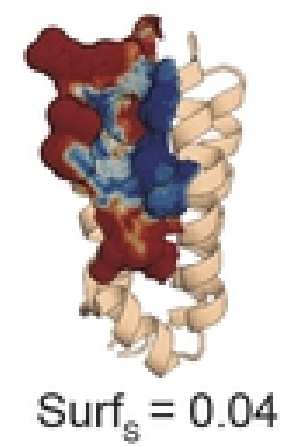

Surf_03

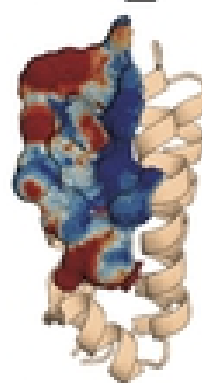

Surf $_{\mathrm{s}}=0.45$
C

\section{RosettaSurf :}

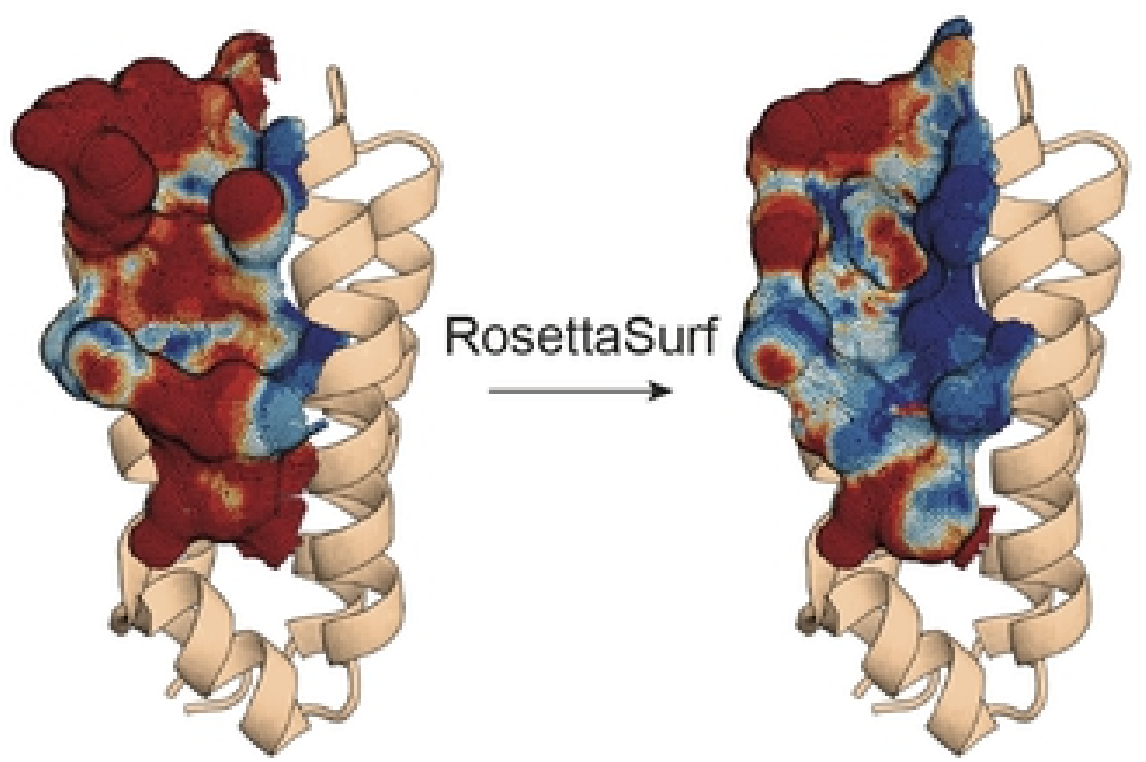

WT scaffold

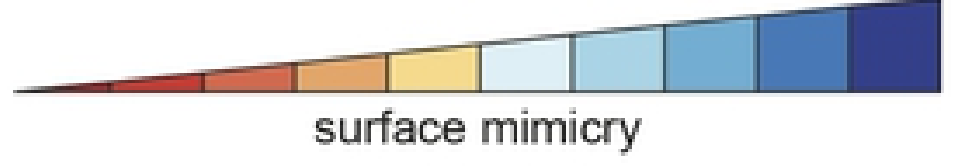

D

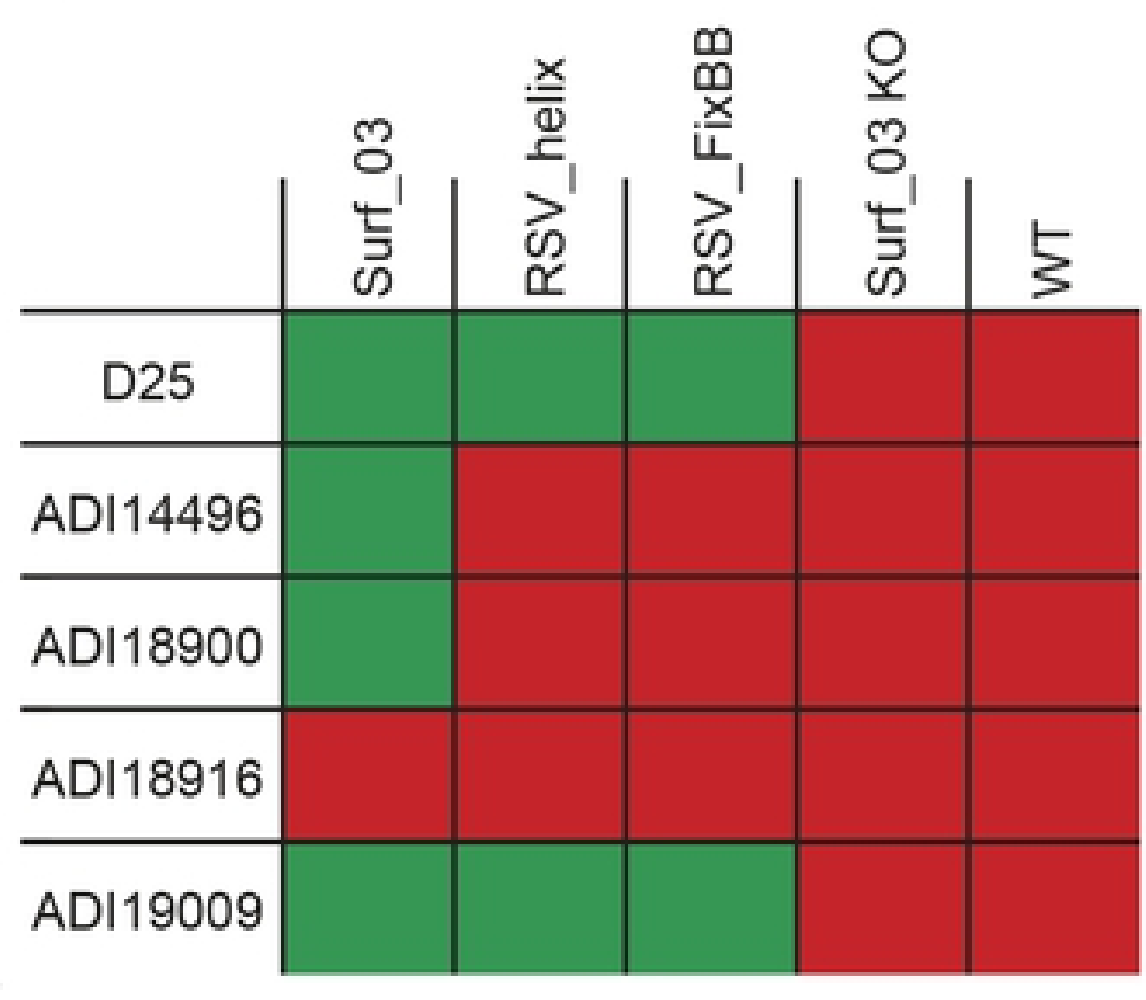

Fig 5

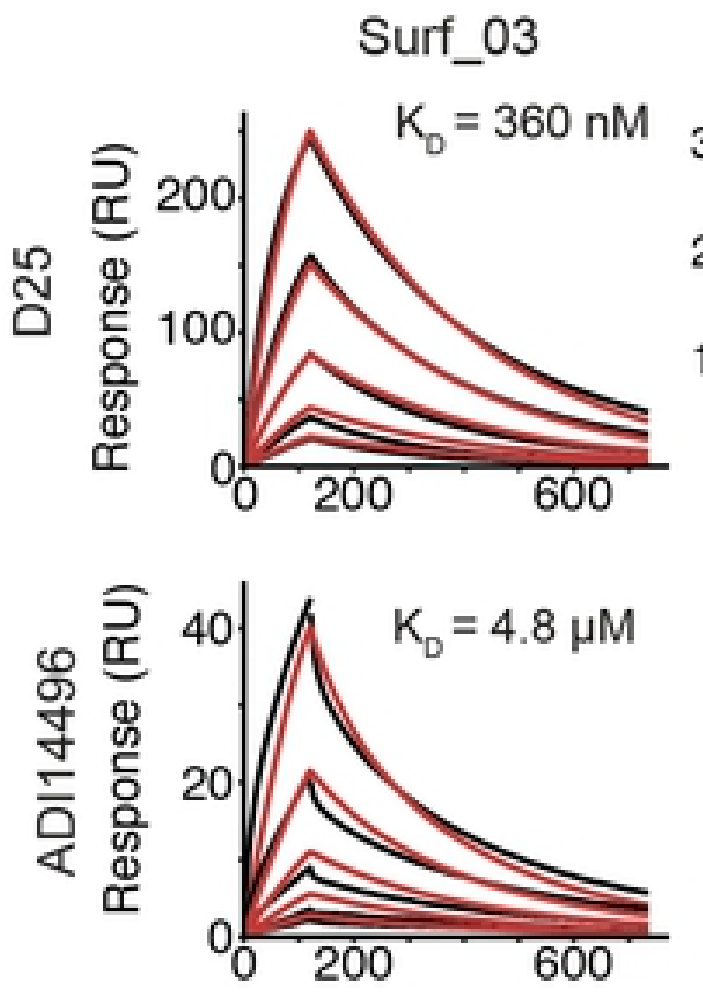

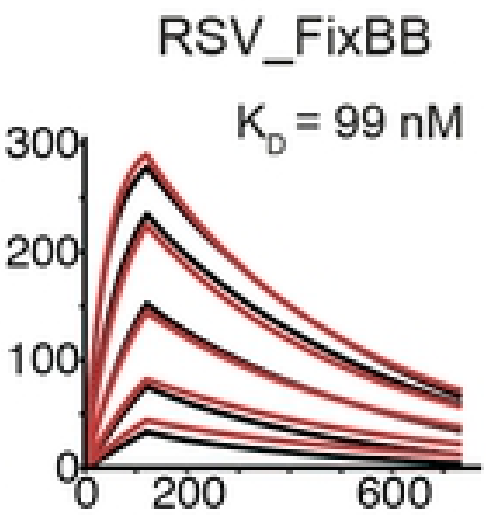
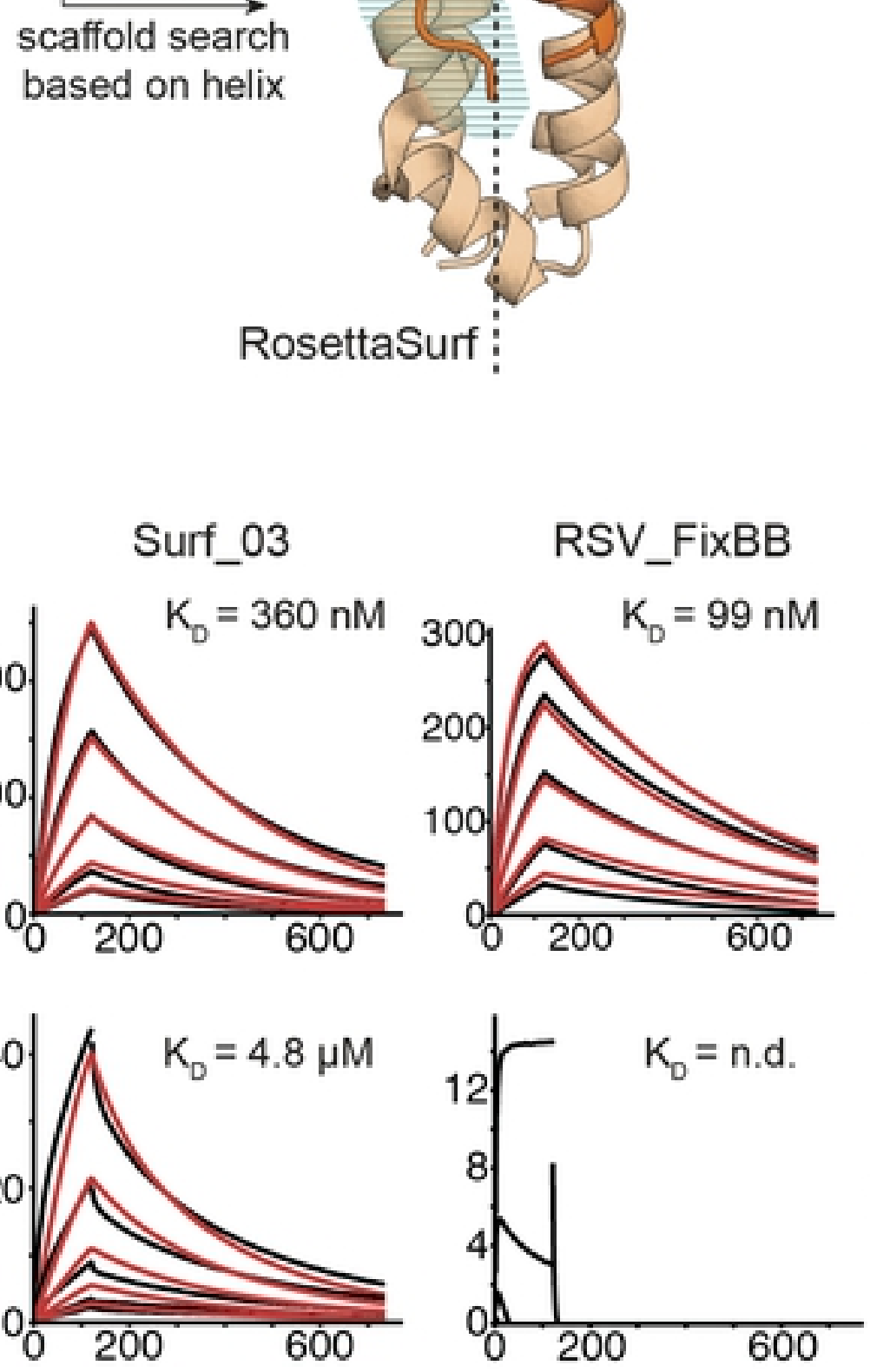
based on helix

\section{Surf_03}

